\title{
Spin Structures and Superstrings
}

\author{
Jacques Distler, Daniel S. Freed, and Gregory W. Moore
}

\begin{abstract}
In superstring theory spin structures are present on both the 2-dimensional worldsheet and 10-dimensional spacetime. We present a new proposal for the $B$-field in superstring theory and demonstrate its interaction with worldsheet spin structures. Our formulation generalizes to orientifolds, where various twistings appear. A special case of the orientifold worldsheet $B$-field amplitude is a $K O$-theoretic construction of the $\mathbb{Z} / 8 \mathbb{Z}$-valued Kervaire invariant on pin $^{-}$surfaces.
\end{abstract}

The Type II superstring in the NSR formulation is a theory of maps from a closed surface $\Sigma$ - the worldsheet-to a 10-manifold $X$-spacetime. The spin structures of the title are present on both the worldsheet and the spacetime. Their roles have been explored in many works; a sampling of references includes [GSO1, GSO2, SS1, SS2, R, SW, DH, AgMV, AgGMV, AW]. In this paper we identify several new phenomena which are intimately related to a new Dirac quantization condition for the $B$-field (Proposal 1.4). For example, in our approach the $B$-field amplitude depends on the worldsheet spin structure. In particular, the distinction between Types IIB and IIA is encoded in the $B$-field and the worldsheet $B$-field amplitude includes the usual signs in the sum over spin structures. In another direction we answer the question: How does the spacetime spin structure impact the worldsheet theory in the lagrangian formulation? It turns up in

To Isadore Singer on the occasion of his 85th birthday

Date: July 17, 2010

Report \#: UTTG-07-10.

The work of J.D. is supported by the National Science Foundation under grant PHY0455649 and a grant from the US-Israel Binational Science Foundation.

The work of D.S.F. is supported by the National Science Foundation under grant DMS-0603964.

The work of G.W.M. is supported by the DOE under grant DE-FG02-96ER40949.

We also thank the Aspen Center for Physics for providing a stimulating environment for many discussions related to this paper.

(C)2011 International Press 
the definition of the partition function of worldsheet fermions, i.e., in computing the pfaffian of the Dirac operator on $\Sigma$. For orientifolds of the Type II superstring, including the Type I superstring, there are several new features. For example, we define precisely the twisted notions of spin structure needed on $\Sigma$ and on $X$. We also consider the worldsheet $B$-field amplitude and the partition function of worldsheet fermions. It turns out that each is anomalous and that these anomalies cancel. That anomaly cancellation is the subject of a future paper [DFM2]; here we are content to motivate that work and consider some special cases.

Evidently, these spin structure considerations are closely tied to the $B$-field $\check{\beta}$, with which we begin in $\S 1$. Quite generally, Dirac quantization of charges and fluxes is implemented by generalized cohomology theories. For the oriented bosonic string the $B$-field has a flux quantized by $H^{3}(X ; \mathbb{Z})$. We locate the superstring $B$-field quantization condition in a generalized cohomology theory $R$ which is a truncation of connective $K O$-theory. Then the $B$-field is modeled in the differential cohomology group $\check{R}^{-1}(X)$ using the general development of differential cohomology in $[\mathbf{H S}]$. In $\S 2$ we take up the integral of $\phi^{*} \breve{\beta}$ on the worldsheet $\Sigma$ for maps $\phi: \Sigma \rightarrow X$. The presence of $K O$-theory suggests the dependence on worldsheet spin structures. We show how the standard $\mathbb{Z} / 2 \mathbb{Z}$-valued quadratic function on spin structures $[\mathbf{A} \mathbf{1}]$ is embedded in the $B$-field amplitude, leading to the distinction between Types IIB and IIA. A generalization of the Scherk-Schwarz construction $[\mathbf{S S 1}, \mathbf{S S 2}]$ is also part of our $B$-field amplitude. Orbifolds (in the sense of string theory) and orientifolds are introduced in $\S 3$. To accommodate the former we allow $X$ to be an orbifold (in the sense of differential geometry); the orientifold is encoded in a double cover $\pi: X_{w} \rightarrow X$ of orbifolds. The $B$-field $\check{\beta}$ is now quantized by the $R$-cohomology of the Borel construction applied to $X$, with local coefficients determined by the double cover $\pi$ (Proposal 3.7). The integral of $\phi^{*} \check{\beta}$ is taken up in $\S 4$. We posit a spin structure on the orientation double cover $\hat{\pi}: \widehat{\Sigma} \rightarrow \Sigma$ of the worldsheet. In case this refines and is refined to a $\operatorname{pin}^{-}$structure the integral of $\phi^{*} \breve{\beta}$ may be easily defined. For a certain universal $B$-field this yields a $K O$-theoretic construction of the $\mathbb{Z} / 8 \mathbb{Z}$-valued Kervaire invariant on pin $^{-}$surfaces $[\mathbf{B r o}],[\mathbf{K T}, \S 3]$. For a general (non-pin ${ }^{-}$) spin structure on $\widehat{\Sigma}$ the $B$-field amplitude is anomalous (4.13); its definition is postponed to [DFM2]. In $\S 5$ we prove a formula for the pfaffian line of the Dirac operator in a related one-dimensional supersymmetric quantum mechanical model, the one which computes the index of the Dirac operator. That formula is a categorified index theorem in low dimensions. We see explicitly how the spin structure on spacetime enters. This result is included here as motivation for [DFM2], where we take up the analogous problem on the two-dimensional worldsheet. The precise nature of the spin structure on spacetime for orientifolds is the subject of $\S 6$. It is a twisted version of the usual notion of spin structure, where the twisting depends on the orientifold double cover $\pi: X_{w} \rightarrow X$ as well as the $B$-field $\check{\beta}$. 
The telegraphic précis [DFM1] outlines many aspects of orientifold theory. This is the first of several papers which expatiate on this résumé. These papers provide motivation, give precise definitions, develop some background mathematics, state and prove the main theorems, and give applications to physics. The geometry of the $B$-field is further developed in subsequent papers. In [DFM2] we build a geometric model of $\check{R}^{-1}(X)$. The geometric model is used in [DFM3] to twist $K$-theory and its cousins, thus defining the home of the Ramond-Ramond field on $X$. The $B$-field is a twisting of $K$-theory. This relation to twistings of $K$-theory is one of the main motivations for the choice of Dirac quantization condition for the $B$-field.

The ideas here touch on many mathematical works of Isadore Singer: among others his recent paper $[\mathbf{H S}]$ on quadratic forms and generalized differential cohomology, his many contributions to index theory and the geometry of Dirac operators, and even his use of frame bundles to express geometric structures on manifolds $[\mathbf{S}]$. Beyond that his prescient recognition 30 years ago of the role that theoretical high energy physics would play in late $20^{\text {th }}$ century and early $21^{\text {st }}$ century mathematics has had enormous influence on the entire field.

We thank Andrew Blumberg, Mike Hopkins, Isadore Singer, and Edward Witten for helpful discussions.

\section{1. $B$-fields and generalized differential cohomology}

In classical physics an abelian gauge field is determined by its field strength $F$, a closed differential form on spacetime $X$. The archetype is the Maxwell electromagnetic field, a closed 2-form in 4 spacetime dimensions. ${ }^{1}$ Abelian gauge theories include an electric current $j$, which in Maxwell theory is a closed 3-form with compact support on spacelike hypersurfaces. The de Rham cohomology class of $F$ is called the classical flux ${ }^{2}$ and the de Rham cohomology class of $j$ the classical charge. (The latter is taken with compact supports in spatial directions.) In quantum theories Dirac's quantization principle constrains these classical fluxes and charges to full lattices inside the appropriate de Rham cohomology groups. For example, the quantum Maxwell electromagnetic flux is constrained to the image of $H^{2}(X ; \mathbb{Z})$ in $H^{2}(X ; \mathbb{R}) \cong H_{d R}^{2}(X)$. It is natural to refine the flux to the abelian group $H^{2}(X ; \mathbb{Z})$. Indeed, in the quantum theory the Maxwell electromagnetic field is modeled as a connection on a principal circle bundle $P \rightarrow X$, and the flux is the topological equivalence class of $P$. The electric charge is then refined to $H^{3}(X ; \mathbb{Z})$ (with appropriate supports), and there is a magnetic charge in the quantum theory as well. This leads to the notion that for any abelian gauge field, charges and fluxes lie in abelian groups which are cohomology groups of spacetime. It is a relatively recent discovery

\footnotetext{
${ }^{1}$ The word 'gauge' in 'classical gauge theory' applies when we identify $\Omega^{2}(X)_{\text {exact }} \cong$ $\Omega^{1}(X) / \Omega^{1}(X)_{\text {closed }}$.

${ }^{2}$ Our usage of 'flux' is not entirely standard.
} 
that generalized cohomology groups may occur. Spacetime anomaly cancellation [GHM, MM] led to the proposal, further elaborated in [W2], that the Ramond-Ramond charges in superstring theory are properly quantized by K-theory, at least in the large distance and weak coupling limit. Similarly, the fluxes are also quantized by K-theory $[\mathbf{F H}, \mathbf{M W}]$. In general, to quantize a classical abelian gauge field one must choose a generalized cohomology group which reproduces the appropriate de Rham cohomology vector space after tensoring over the reals. The choice of cohomology theory is an input. There are many physical considerations which motivate the choice and can be used to justify it. See $[\mathbf{F 1}$, Part 3], [W3, OS, M] for leisurely expositions of these ideas, including some examples.

In string theory, spacetime $X$ is a smooth manifold whose dimension is 26 for the bosonic string and 10 for the superstring. ${ }^{3}$ In each case there is an abelian gauge field - the " $B$-field" - whose field strength is a closed 3 -form $H \in \Omega^{3}(X)$. Dirac's principle applies and we must locate the quantum flux in a cohomology group. The most natural choice applies a simple degree shift to the Maxwell case.

Supposition 1.1. The flux of the oriented bosonic string B-field lies in $H^{3}(X ; \mathbb{Z})$.

This supposition is certainly well-established $[\mathbf{R W}]$. In this section we make a new proposal for the oriented superstring.

1.1. The cohomology theory $\boldsymbol{R}$. Let $k o$ denote connective $K O$ theory. One construction [Se] starts with the symmetric monoidal category of real vector spaces and applies a de-looping machine to construct an infinite loop structure on its classifying space. More concretely, $k o$ is the real version of $K$-theory developed in [A2] before inverting the Bott element; for any space $M$ the abelian groups $k o^{q}(M)$ vanish for $q>0$ and $k o^{-q}(M) \cong K O^{-q}(M)$ for $q \geq 0$. Define the Postnikov truncation ${ }^{4}$

$$
R:=k o\langle 0 \cdots 4\rangle \text {. }
$$

Then $R$ is a generalized multiplicative cohomology theory, more precisely an $E^{\infty}$-ring spectrum. Its nonzero homotopy groups are

$$
\left\{\pi_{0}, \pi_{1}, \pi_{2}, \pi_{3}, \pi_{4}\right\}(R) \cong\{\mathbb{Z}, \mathbb{Z} / 2 \mathbb{Z}, \mathbb{Z} / 2 \mathbb{Z}, 0, \mathbb{Z}\},
$$

a truncated Bott song. These are also the nonzero $R$-cohomology groups of a point and they occur in nonpositive degrees, as $R^{-q}(\mathrm{pt})=\pi_{q}(R)$. If we represent the theory as a (loop) spectrum $\left\{R_{p}\right\}_{p \in \mathbb{Z}}$, so that for any space $M$

\footnotetext{
${ }^{3}$ We use 'superstring' as a shorthand for 'Type II superstring' in a sigma model formulation.

${ }^{4}$ We use the version of Postnikov truncation for connective $E^{\infty}$-ring spectra [B]. The notation ' $R$ ' for a multiplicative spectrum is generic, ergo uninformative, but it would be cumbersome to use ' $k o\langle 0 \cdots 4\rangle$ ' instead.
} 
and $q \geq 0$ we compute $R^{-q}(M)=\left[M, R_{-q}\right]$ as the abelian group of homotopy classes of maps into the space $R_{-q}$, then (1.3) are the homotopy groups of the space $R_{0}$.

Here is our new proposal for the $B$-field in superstring theory. Let $X$ be a smooth 10-dimensional manifold which plays the role of spacetime in the superstring.

Proposal 1.4. The flux of the oriented superstring B-field $\check{\beta}$ lies in $R^{-1}(X)$.

As a first check we note that the nonzero homotopy groups of the space $R_{-1}$ are

$$
\left\{\pi_{0}, \pi_{1}, \pi_{2}, \pi_{3}\right\}\left(R_{-1}\right) \cong\{\mathbb{Z} / 2 \mathbb{Z}, \mathbb{Z} / 2 \mathbb{Z}, 0, \mathbb{Z}\}
$$

so after tensoring with the reals we obtain the Eilenberg-MacLane space $K(\mathbb{R}, 3)$ which computes real cohomology in degree 3 . This is as it should be: the classical fluxes of the classical field $H$ lie in the degree 3 de Rham cohomology of the manifold $X$. We explore some physical consequences of the nonzero torsion homotopy groups in $\S 2$.

We record the exact sequence of abelian groups

$$
0 \longrightarrow H^{3}(M ; \mathbb{Z}) \longrightarrow R^{-1}(M) \stackrel{(t, a)}{\longrightarrow} H^{0}(M ; \mathbb{Z} / 2 \mathbb{Z}) \times H^{1}(M ; \mathbb{Z} / 2 \mathbb{Z}) \longrightarrow 0
$$

which follows from the Postnikov tower (see (1.5)) and holds for any space $M$. There is not a corresponding exact sequence of cohomology theories; the $k$-invariant between the bottom two homotopy groups is nonzero. The quotient group in (1.6) is more properly regarded as the group of equivalence classes of $\mathbb{Z} / 2 \mathbb{Z}$-graded real line bundles (equivalently: $\mathbb{Z} / 2 \mathbb{Z}$-graded double covers) over $M$. The exact sequence (1.6) immediately implies

$$
R^{-1}(\mathrm{pt}) \cong \mathbb{Z} / 2 \mathbb{Z}
$$

and we can identify a generator with the nonzero element $\eta \in k o^{-1}(\mathrm{pt}) \cong$ $K O^{-1}(\mathrm{pt}) \cong \mathbb{Z} / 2 \mathbb{Z}$.

There is a natural splitting of (1.6) as sets (not as abelian groups). To construct it we interpret the quotient group as the group of $\mathbb{Z} / 2 \mathbb{Z}$-graded real line bundles and apply the following lemma.

LemMA 1.8. Let $V \rightarrow M$ be a real vector bundle over a space $M$ and $[V] \in R^{0}(M)$ its equivalence class under the map $k o^{0}(M) \rightarrow R^{0}(M)$. Then for $\eta[V] \in R^{-1}(M)$ we have

$$
\begin{aligned}
& t(\eta[V])=\operatorname{rank}(V) \quad(\bmod 2) \\
& a(\eta[V])=w_{1}(V),
\end{aligned}
$$

where $\operatorname{rank}(V): \pi_{0} M \rightarrow \mathbb{Z}$ is the rank. 
Proof. The map $t$ in (1.6) is determined on the 0-skeleton $M^{0}$ of $M$, and $V$ is equivalent to $\operatorname{rank}(V)$ in $k o^{0}\left(M^{0}\right)$. This reduces (1.9) to the assertion $t(\eta)=1$, which is essentially the isomorphism (1.7). The map $a$ in (1.6) is determined on the 1-skeleton, and as $a(\eta)=0$ we can replace $V$ by its reduced determinant line bundle Det $V-1$, which is equivalent to $V-\operatorname{rank} V$ in the reduced group $\widetilde{k o}^{0}\left(M^{1}\right)$. Hence it suffices to prove (1.10) for the universal real line bundle $L^{\text {univ }} \rightarrow \mathbb{R P}^{\infty}$. Identify $k o^{-1}(\mathrm{pt}) \cong \widetilde{k o}^{0}\left(\mathbb{R P}^{1}\right)$ and represent $\eta$ by the reduced Möbius line bundle $(H-1) \rightarrow \mathbb{R P}^{1}$. Then $\eta\left[L^{\text {univ }}\right]$ is represented by the external tensor product $(H-1) \otimes L^{\text {univ }} \rightarrow \mathbb{R P}^{1} \times$ $\mathbb{R} \mathbb{P}^{\infty}$. To compute the $a$-component in (1.6) we restrict to the 1 -skeleton $\mathbb{R P}^{1} \subset \mathbb{R} \mathbb{P}^{\infty}$, over which $L^{\text {univ }}$ is identified with $H$. Again since $a(\eta)=0$ we may pass to $(H-1) \otimes(H-1) \rightarrow \mathbb{R P}^{1} \times \mathbb{R P}^{1}$, and this represents $\eta^{2} \in k o^{-2}(\mathrm{pt})$, which is nonzero. This proves $\eta[H-1]$ is the nonzero class in $R^{-1}\left(\mathbb{R P}^{1} / \mathbb{R P}^{0}\right) \cong H^{1}\left(\mathbb{R P}^{1} / \mathbb{R P}^{0} ; \mathbb{Z} / 2 \mathbb{Z}\right)$. Therefore $a(\eta[H-1])$, hence also $a\left(\eta\left[L^{\text {univ }}\right]\right)$, is nonzero.

1.2. Generalized differential cohomology and superstring $\boldsymbol{B}$-fields. Semi-classical models of abelian gauge fields, which appear as background fields or as inputs to a functional integral, combine the local information of the classical field strength with the integrality of the quantum flux. As mentioned earlier the model for the Maxwell field is a circle bundle with connection: its curvature is the classical field strength and its Chern class the quantum flux. Notice that there are nontrivial connections for which both of these vanish. In other words, the combination of classical field strength and quantum flux do not determine the semi-classical gauge field. Equivalence classes of Maxwell fields, thus of circle connections, on any smooth manifold $M$ form an infinite dimensional abelian Lie group $\operatorname{Pic}_{\nabla}(M)$, a differential-geometric analog of the Picard group in algebraic geometry. Its group of path components is

$$
\pi_{0} \operatorname{Pic}_{\nabla}(M) \cong H^{2}(M ; \mathbb{Z})
$$

the group of equivalence classes of circle bundles. The map $\operatorname{Pic}_{\nabla}(M) \rightarrow$ $\pi_{0} \operatorname{Pic}_{\nabla}(M)$ forgets the connection. The torus $H^{1}(M ; \mathbb{Z}) \otimes \mathbb{R} / \mathbb{Z}$ of equivalence classes of flat connections on the trivial circle bundle acts freely on the identity component $\operatorname{Pic}_{\nabla}^{0}(M)$ by tensor product, and the quotient

$$
\operatorname{Pic}_{\nabla}^{0}(M) \rightarrow \Omega_{\text {exact }}^{2}(M)
$$

is the vector space of exact 2-forms. Other components of $\operatorname{Pic}_{\nabla}(M)$ are also total spaces of principal $H^{1}(M ; \mathbb{Z}) \otimes \mathbb{R} / \mathbb{Z}$ bundles; the bases are affine translates of $\Omega_{\text {exact }}^{2}(M)$ in the topological vector space of closed 2-forms, affine spaces of closed forms with a fixed de Rham cohomology class in the lattice Image $\left(H^{2}(M ; \mathbb{Z}) \rightarrow H^{2}(M ; \mathbb{R})\right)$.

Cheeger-Simons $[\mathbf{C S}]$ introduced abelian Lie groups $\check{H}^{q}(M)$ for all integers $q$ which generalize $\breve{H}^{2}(M) \cong \operatorname{Pic}_{\nabla}(M)$. The group $\check{H}^{1}(M)$ is the group 
of smooth maps $M \rightarrow \mathbb{T}$ into the circle group. The group $\check{H}^{3}(M)$ may be modeled as equivalence classes of $\mathbb{T}$-gerbes with connection or bundle gerbes $[\mathbf{B r}, \mathbf{H i}, \mathbf{M u}]$. The original definition of $\check{H}^{q}(M)$ is in terms of the integral over smooth singular $(q-1)$-cycles, generalizing the holonomy of a $\mathbb{T}$-connection around a loop. There is an alternative approach using sheaves, modeled after a construction of Deligne in algebraic geometry. Hopkins-Singer $[\mathbf{H S}]$ provide two important supplements. First, they define differential cohomology groups $\check{h}^{\bullet}(M)$ for any cohomology theory $h$. Second, they define spaces ${ }^{5} \check{h}_{p}(M)$ such that $\pi_{0} \check{h}_{p}(M) \cong \check{h}^{p}(M)$. Thus points of $\check{h}_{p}(M)$ may be considered as geometric objects whose equivalence class lies in $\check{h}^{p}(M)$, just as a circle bundle with connection has an equivalence class in $\operatorname{Pic}_{\nabla}(M)$. For the specific cohomology theory $R$ in (1.2) fix a singular cocycle $\iota \in C^{3}\left(R_{-1} ; \mathbb{R}\right)$ whose cohomology class is a normalized generator of $H^{3}\left(R_{-1} ; \mathbb{R}\right)$. Then a point of degree -1 is a triple $(c, h, \omega)$, where

$$
\begin{aligned}
& c: M \longrightarrow R_{-1} \\
& h \in C^{2}(M ; \mathbb{R}) \\
& \omega \in \Omega^{3}(M)
\end{aligned}
$$

and $h$ satisfies $\delta h=\omega-c^{*} \iota$. (It follows that $d \omega=0$.) We give $\check{R}^{p}(M)$ the structure of an abelian Lie group for which

$$
\pi_{0} \check{R}^{p}(M) \cong R^{p}(M)
$$

and each component is a principal $R^{p-1}(M ; \mathbb{R} / \mathbb{Z})$-bundle over an affine space of closed differential forms.

The preceding discussion leads to corollaries of Supposition 1.1 and Proposal 1.4:

The oriented bosonic string $B$-field $\check{\beta}$ is a point in $\check{H}_{3}(X)$.

The oriented superstring $B$-field $\check{\beta}$ is a point in $\check{R}_{-1}(X)$.

In [DFM2] we give a concrete differential-geometric model of the superstring $B$-field, whereas the model in terms of the spaces $\check{R}_{p}(X)$ is more homotopy-theoretic. In any case for the purposes of this paper we only need the equivalence class $[\check{\beta}] \in \check{R}^{-1}(X)$ of $\check{\beta}$. We remark that $\check{\beta}$ determines $\beta \in R_{-1}$ whose equivalence class is $[\beta] \in R^{-1}(X)$; see (1.19) below. Then using (1.6) we define

$$
(t(\check{\beta}), a(\check{\beta})) \in H^{0}(X ; \mathbb{Z} / 2 \mathbb{Z}) \times H^{1}(X ; \mathbb{Z} / 2 \mathbb{Z}) .
$$

The physical significance of (1.17) is explained in subsequent sections.

We record the following exact sequences, which are specializations to the case at hand of general facts about differential cohomology and hold for any

\footnotetext{
${ }^{5}$ In fact, they define simplicial sets. We use the moniker 'points' for its 0-simplices.
} 
smooth manifold $M$ :

$$
\begin{array}{r}
0 \longrightarrow R^{-(q+1)}(M ; \mathbb{R} / \mathbb{Z}) \longrightarrow \check{R}^{-q}(M) \longrightarrow \Omega_{\mathbb{Z}}^{4-q}(M) \longrightarrow 0 \\
0 \longrightarrow \Omega^{3-q}(M) / \Omega_{\mathbb{Z}}^{3-q}(M) \longrightarrow \check{R}^{-q}(M) \longrightarrow R^{-q}(M) \longrightarrow 0
\end{array}
$$

Here $q=1,2,3$ and $\Omega_{\mathbb{Z}}^{4-q}(M)$ denotes the space of closed forms with integral periods. In particular, it follows from these sequences and (1.7) that

$$
R^{-2}(\mathrm{pt} ; \mathbb{R} / \mathbb{Z}) \cong \check{R}^{-1}(\mathrm{pt}) \cong R^{-1}(\mathrm{pt}) \cong \mathbb{Z} / 2 \mathbb{Z} .
$$

The nonzero element $\check{\eta}$ of (1.20) pulls back to any $M$ and is a special $B$-field in oriented superstring theory. It may be identified with the generator of $k o^{-2}(\mathrm{pt} ; \mathbb{R} / \mathbb{Z}) \cong K O^{-2}(\mathrm{pt} ; \mathbb{R} / \mathbb{Z}) \cong \mathbb{Z} / 2 \mathbb{Z}$. Of course, $\check{\eta}$ maps to $\eta$ under the Bockstein homomorphism $R^{-2}(\mathrm{pt} ; \mathbb{R} / \mathbb{Z}) \rightarrow R^{-1}(\mathrm{pt} ; \mathbb{Z})$.

Any real line bundle $L \rightarrow M$ determines

$$
\check{\eta}[L] \in R^{-2}(M ; \mathbb{R} / \mathbb{Z}) \longrightarrow \check{R}^{-1}(M)
$$

with $t(\check{\eta}[L])=1$ and $a(\check{\eta}[L])=w_{1}(L)$; see Lemma 1.8.

REMARK 1.22. An oriented superstring spacetime $X^{10}$ is endowed with a spin structure $\kappa$. (See $\S 2.1$ for a review of spin structures. The twisted notion of spin structure for superstring orientifold spacetimes is the subject of $\S 6$.) Now the $B$-field $\check{\beta}$ may be written (Lemma 1.8) as a sum of an object $\check{\beta}_{0}$ in $\check{H}^{3}(X)$ and a $\mathbb{Z} / 2 \mathbb{Z}$-graded double cover $K \rightarrow X$, the latter with characteristic class $(t(\check{\beta}), a(\check{\beta})) \in H^{0}(X ; \mathbb{Z} / 2 \mathbb{Z}) \times H^{1}(X ; \mathbb{Z} / 2 \mathbb{Z})$. We can shuffle the data: Define two spin structures $\kappa_{\ell}=\kappa, \kappa_{r}=\kappa+K$ on spacetime and consider the $B$-field to be $\check{\beta}_{0}$. The two spin structures then correlate with the two spin structures $\alpha_{\ell}, \alpha_{r}$ on the worldsheet; see Definition 2.4 below. This splitting into 'left' and 'right' does not generalize to orientifolds.

\section{The $B$-field amplitude and worldsheet spin structures}

The spacetime for oriented bosonic string theory is a smooth 26-manifold $X$, and the $B$-field $\check{\beta}$ has an equivalence class in $\check{H}^{3}(X)$; see Supposition 1.1. The worldsheet in oriented bosonic string theory is a closed 2-manifold $\Sigma$ with orientation $\mathfrak{o}$ and a smooth map $\phi: \Sigma \rightarrow X$. (It represents the propagation of closed strings; for open strings $\Sigma$ may have a boundary.) One factor in the exponentiated action of the worldsheet theory is

$$
\exp \left(2 \pi i \int_{\Sigma} \phi^{*} \check{\beta}\right)
$$

it only depends on the equivalence class $[\check{\beta}] \in \check{H}^{3}(X)$ and is defined using the pushforward in ordinary differential cohomology: $\phi^{*}[\check{\beta}] \in \check{H}^{3}(\Sigma)$ and the orientation $\mathfrak{o}$ on $\Sigma$ determines a pushforward map [HS, §3.5]

$$
\int_{(\Sigma, \mathfrak{o})}: \check{H}^{3}(\Sigma) \longrightarrow \check{H}^{1}(\mathrm{pt}) \cong \mathbb{R} / \mathbb{Z}
$$


In this section we define the analog for the superstring and explore some consequences.

2.1. Spin structures on superstring worldsheets. As a preliminary we quickly review spin structures. Recall that the intrinsic geometry of a smooth $n$-manifold $M$ is encoded in its principal $G L_{n} \mathbb{R}$-bundle of frames $\mathcal{B}(M) \rightarrow M$. A point of $\mathcal{B}(M)$ is a linear isomorphism $\mathbb{R}^{n} \rightarrow T_{m} M$ for some $m \in M$. Choose a Riemannian metric on $M$, equivalently, a reduction to an $O_{n}$-bundle of frames $\mathcal{B}_{O}(M) \rightarrow M$. The spin group

$$
\rho: \operatorname{Spin}_{n} \longrightarrow O_{n}
$$

is the double cover of the index two subgroup $S O_{n} \subset O_{n}$. A spin structure on $M$ is a principal $\operatorname{Spin}_{n}$-bundle $\mathcal{B}_{\text {Spin }} \rightarrow M$ together with an isomorphism of the associated $O_{n}$-bundle with $\mathcal{B}_{O}(M)$. It induces an orientation on $M$ via the cover $\operatorname{Spin}_{n} \rightarrow S O_{n}$. The space of Riemannian metrics is contractible, so a spin structure is a topological choice and can alternatively be described in terms of a double cover of an index two subgroup of $G L_{n} \mathbb{R}$. An isomorphism of spin structures is a map $\mathcal{B}_{\text {Spin }} \rightarrow \mathcal{B}_{\text {Spin }}^{\prime}$ such that the induced map on $O_{n}$-bundles commutes with the isomorphisms to $\mathcal{B}_{O}(M)$. The opposite spin structure to $\mathcal{B}_{\text {Spin }} \rightarrow M$ is the complement of $\mathcal{B}_{\text {Spin }}$ in the principal $\operatorname{Pin}_{n}^{-}$-bundle associated to the inclusion $\operatorname{Spin}_{n} \hookrightarrow \operatorname{Pin}_{n}^{-}$; see [KT, Lemma 1.9] for more elaboration. ${ }^{6}$ If $M$ admits spin structures, then the collection of spin structures forms a groupoid whose set of equivalence classes $\mathcal{S}(M)$ is a torsor for $H^{0}(M ; \mathbb{Z} / 2 \mathbb{Z}) \times H^{1}(M ; \mathbb{Z} / 2 \mathbb{Z})$; the action of a function $\delta: \pi_{0} M \rightarrow \mathbb{Z} / 2 \mathbb{Z}$ in $H^{0}(M ; \mathbb{Z} / 2 \mathbb{Z})$ sends a spin structure to its opposite on components where $\delta=1$ is the nonzero element. The automorphism group of any spin structure is isomorphic to $H^{0}(M ; \mathbb{Z} / 2 \mathbb{Z})$; a function $\delta: \pi_{0} M \rightarrow \mathbb{Z} / 2 \mathbb{Z}$ acts by the central element of $\operatorname{Spin}_{n}$ on components where $\delta=1$. The manifold $M$ admits spin structures if and only if the Stiefel-Whitney classes $w_{1}(M), w_{2}(M)$ vanish.

A superstring worldsheet $(\Sigma, \mathfrak{o})$ is oriented and is equipped with a pair of spin structures ${ }^{7} \alpha_{\ell}, \alpha_{r}$ which induce opposite orientations at each point. Our convention is that the left spin structure $\alpha_{\ell}$ induces the chosen orientation $\mathfrak{o}$. Observe that a spin structure is local and can be considered as a field in the sense of physics. It is a discrete field, in fact a finite field on a compact manifold: there are only finitely many spin structures up to isomorphism. As with gauge fields, spin structures have automorphisms so there is a groupoid of fields rather than a space of fields.

DeFinition 2.4. The topological data on an oriented superstring worldsheet $(\Sigma, \mathfrak{o})$ is a discrete field $\alpha$ which on each connected orientable open

\footnotetext{
${ }^{6}$ Recall that $\operatorname{Pin}_{n}^{ \pm}$sits in the Clifford algebra Cliff $_{n}^{ \pm}$whose generators satisfy $\gamma^{2}= \pm 1$. Either sign can be used to construct the opposite spin structure.

7 ' $\ell$ ' and ' $r$ ' stand for 'left' and 'right'.
} 
set $U \subset \Sigma$ is a pair of spin structures which induce opposite orientations of $U$.

In more detail, this is the indicated data on each connected orientable open set, isomorphisms of the spin structures on intersections of such open sets, and a coherence condition among the isomorphisms on triple intersections. The global orientation $\mathfrak{o}$ is used to construct from $\alpha$ a global spin structure $\alpha_{\ell}$ which induces $\mathfrak{o}$ and a spin structure $\alpha_{r}$ which induces the opposite orientation $-\mathfrak{o}$. The global spin structures $\alpha_{\ell}, \alpha_{r}$ need not be opposites (as defined in the previous paragraph). For orientifold models (§3) the worldsheet does not have a global orientation, indeed may be nonorientable, but it retains the discrete field $\alpha$; see Definition 4.8. In string theory one integrates over $\alpha$, i.e., sums over the spin structures.

REMARK 2.5. We could, of course, replace $\alpha$ in Definition 2.4 with the pair of spin structures $\alpha_{\ell}, \alpha_{r}$. Our formulation emphasizes both the local nature of the spin structure and that the same local field is present on worldsheets in orientifold superstring theories.

2.2. Superstring $B$-field amplitudes. Let $X$ be a 10 -manifold-a superstring spacetime - and $\breve{\beta}$ a $B$-field on $X$ as defined in (1.16). We define the oriented superstring $B$-field amplitude (2.1), which only depends on the equivalence class $[\check{\beta}] \in \check{R}^{-1}(X)$. To do so we replace (2.2) with a pushforward in differential $R$-theory. The main point is that the cohomology theory $R$ is Spin-oriented, that is, there is a pushforward in topological $R$-theory on spin manifolds. It is the Postnikov truncation of the pushforward in ko-theory defined from the spin structure (which by the Atiyah-Singer index theorem has an interpretation as an index of a Dirac operator). In fact, because we are in sufficiently low dimensions we can identify it exactly with the pushforward in $k o$, a fact which is useful in the proof of the Theorem 2.9 below. Combining with integration of differential forms we obtain a pushforward [HS, §4.10]

$$
\int_{\Sigma, \alpha_{\ell}}: \check{R}^{-1}(\Sigma) \longrightarrow \check{R}^{-3}(\mathrm{pt}) \cong \mathbb{R} / \mathbb{Z}
$$

in differential $R$-theory defined using the spin structure $\alpha_{\ell}$ on $\Sigma$. (Use (1.18) to see the isomorphism $\check{R}^{-3}(\mathrm{pt}) \cong \mathbb{R} / \mathbb{Z}$.) This completes the definition of the $B$-field amplitude. In the remainder of this section we investigate special cases which go beyond the $B$-field amplitude for the oriented bosonic string.

Let $(\Sigma, \mathfrak{o})$ be a closed oriented surface and $\mathcal{S}(\Sigma, \mathfrak{o})$ the set of equivalence classes of spin structures which refine the given orientation. Note $\mathcal{S}(\Sigma, \mathfrak{o})$ is a torsor for $H^{1}(\Sigma ; \mathbb{Z} / 2 \mathbb{Z})$. Let

$$
q: \mathcal{S}(\Sigma, \mathfrak{o}) \longrightarrow \mathbb{Z} / 2 \mathbb{Z}
$$

be the affine quadratic function which distinguishes even and odd spin structures. It dates back to Riemann and is the Kervaire invariant in dimension 
two; see [HS, §1] for some history. The characteristic property of the quadratic function $q$ is

$$
\begin{aligned}
& q\left(\alpha+a_{1}+a_{2}\right)-q\left(\alpha+a_{1}\right)-q\left(\alpha+a_{2}\right)+q(\alpha)=a_{1} \cdot a_{2}, \\
& \alpha \in \mathcal{S}(\Sigma, \mathfrak{o}), \quad a_{1}, a_{2} \in H^{1}(\Sigma ; \mathbb{Z} / 2 \mathbb{Z}),
\end{aligned}
$$

where $a_{1} \cdot a_{2} \in \mathbb{Z} / 2 \mathbb{Z}$ is the $\bmod 2$ intersection pairing.

TheOrem 2.9. Let $\check{\eta}$ be the nonzero universal B-field in (1.20). For any superstring worldsheet $\phi: \Sigma \rightarrow X$, the B-field amplitude is $(-1)^{q\left(\alpha_{\ell}\right)}$.

This demonstrates that the $B$-field amplitude (2.1) is sensitive to the worldsheet spin structure.

Proof. Let $p: \Sigma \rightarrow$ pt and $p_{*}^{\alpha_{\ell}}: k o^{0}(\Sigma ; \mathbb{Z}) \rightarrow k o^{-2}(\mathrm{pt} ; \mathbb{Z})$ the pushforward (2.6) defined using the spin structure $\alpha_{\ell}$. . Since [HS, §4.10] pushforward is compatible with the exact sequence (1.18), we use push-pull to compute the integral in (2.1) as

$$
p_{*}^{\alpha_{\ell}} p^{*} \check{\eta}=\check{\eta} p_{*}^{\alpha_{\ell}}(1)
$$

The main theorem in $[\mathbf{A 1}]$ states that $p_{*}^{\alpha_{\ell}}(1)=q\left(\alpha_{\ell}\right) \eta^{2}$, where $\eta^{2} \in k o^{-2}(\mathrm{pt}$; $\mathbb{Z}) \cong \mathbb{Z} / 2 \mathbb{Z}$ is the generator. Finally, $\check{\eta} \cdot \eta^{2} \in k o^{-4}(\mathrm{pt} ; \mathbb{R} / \mathbb{Z}) \cong \mathbb{R} / \mathbb{Z}$ is the nonzero element $1 / 2$ of order two [FMS, Proposition B.4].

The space of fields $\mathcal{F}$ in the worldsheet formulation has many components, distinguished by the equivalence class of the spin structures $\alpha$, the homotopy class of $\phi: \Sigma \rightarrow X$, etc. If $\breve{\beta}$ is any $B$-field on $X$, then Theorem 2.9 implies that the theory with $B$-field $\check{\beta}+\check{\eta}$ differs only by the $\operatorname{sign}(-1)^{q\left(\alpha_{\ell}\right)}$ on components of $\mathcal{F}$ with spin structure $\alpha_{\ell}$. Note that $t(\check{\beta}+\check{\eta})=t(\check{\beta})+1$. Recall the notation in (1.17).

Definition 2.11. An oriented superstring has Type IIB on components of $X$ on which $t(\check{\beta}): \pi_{0} X \rightarrow \mathbb{Z} / 2 \mathbb{Z}$ vanishes and has Type IIA on components of $X$ on which $t(\check{\beta})$ is nonzero.

REMARK 2.12. In the Hamiltonian formulation the distinction between Type IIA and Type IIB is a sign in the GSO projection. In the Lagrangian formulation this sign is manifested by the sign $(-1)^{q\left(\alpha_{\ell}\right)}$ in the sum over spin structures $[\mathbf{S W}]$. Also, since the set of isomorphism classes of $B$-fields is an abelian group there is a distinguished element, namely zero. In this sense our approach favors Type IIB as more "fundamental" than Type IIA.

Next, we consider the worldsheet amplitude for the special flat $B$-fields defined in (1.21). 
THEOREM 2.13. Let $L \rightarrow X$ be a real line bundle and $\check{\eta} L$ the corresponding B-field. For a superstring worldsheet $\phi: \Sigma \rightarrow X$, the B-field amplitude is $(-1)^{q\left(\alpha_{\ell}+\phi^{*} L\right)}$.

Proof. We proceed as in the proof of Theorem 2.9. The right hand side of $(2.10)$ is now $\check{\eta} p_{*}\left[\phi^{*} L\right]$. Conclude by observing that the pushforward of $\left[\phi^{*} L\right]$ in the spin structure $\alpha_{\ell}$ is equal to the pushforward of 1 in the spin structure $\alpha_{\ell}+\phi^{*} L$.

Lemma 1.8 implies that $t(\check{\eta}[L])=1$ and $a(\check{\eta}[L])=w_{1}(L)$. We can consider instead the $B$-field $\check{\eta}(L-1)$ for which $t=0$ and $a$ is as before; then combine Theorem 2.9 and Theorem 2.13 to compute the $B$-field amplitude

$$
(-1)^{q\left(\alpha_{\ell}+\phi^{*} L\right)-q\left(\alpha_{\ell}\right)}
$$

for the $B$-field $\check{\eta}(L-1)$.

\section{Orbifolds and orientifolds}

In this section we take up two important variations of the basic Type II superstring. First, suppose a finite group $\Gamma$ acts on a smooth 10 -manifold $Y$. Then there is a superstring theory - the orbifold - whose spacetime is constructed from the pair $(Y, \Gamma)$ by "gauging" the symmetry group $\Gamma$. The main new feature is the inclusion of twisted sectors $[\mathbf{D H V W}]$ : in addition to strings $\phi: S^{1} \rightarrow Y$ one considers for each $\gamma \in \Gamma$ maps $\phi: \mathbb{R} \rightarrow Y$ such that $\phi(s+1)=\gamma \cdot \phi(s)$ for all $s \in \mathbb{R}$. The analog for surfaces is a bit more complicated. Twisted sectors are labeled by a principal $\Gamma$-bundle $P \rightarrow \Sigma$ over a superstring worldsheet $\Sigma$, and then a map to spacetime is a $\Gamma$-equivariant map $\tilde{\phi}: P \rightarrow Y$. If $\tilde{\phi}^{\prime}: P^{\prime} \rightarrow Y$ is another orbifold worldsheet, then a morphism $\tilde{\phi} \rightarrow \tilde{\phi}^{\prime}$ is an isomorphism $P \rightarrow P^{\prime}$ of principal $\Gamma$-bundles which intertwines $\tilde{\phi}, \tilde{\phi}^{\prime}$. The space of these fields is an infinite-dimensional groupoid.

Points of $Y$ connected by elements of $\Gamma$ represent the same points of spacetime $-\Gamma$ is a gauge symmetry - so it is natural to take spacetime as the quotient $Y / / \Gamma$. We keep track of isotropy subgroups, due to non-identity elements $\gamma \in \Gamma$ and $y \in Y$ with $\gamma \cdot y=y$. Now an old construction in differential geometry $[\mathbf{S a}]$, also dubbed [Th] 'orbifold', does exactly that. Furthermore, we can admit as spacetimes orbifolds $X$ which are not global quotients by finite groups, thus widening the collection of models introduced in the previous paragraph. Orbifolds are presented by a particular class of groupoids ${ }^{8}[\mathbf{A L R}]$, a special case being the presentation of a global quotient $X=Y / / \Gamma$ by the pair $(Y, \Gamma)$. We take up groupoid presentations in subsequent papers, but here simply work directly with $X$. A worldsheet is then a map $\phi: \Sigma \rightarrow X$ of orbifolds, and the infinite-dimensional orbifold of

\footnotetext{
${ }^{8}$ We could write 'orbifold'='smooth Deligne-Mumford stack', smooth understood as in 'smooth manifold'.
} 
such maps includes twisted sectors. The reader unfamiliar with differentialgeometric orbifolds may prefer to consider only global quotients $Y / / \Gamma$ and work equivariantly on $Y$.

3.1. Equivariant cohomology and orbifold $\boldsymbol{B}$-fields. There are many extensions of a given cohomology theory $h$ to an equivariant cohomology theory for spaces $Y$ with the action of a compact Lie group $G$. The simplest is the Borel construction. It attaches to $(Y, G)$ the space $Y_{G}=E G \times{ }_{G} Y$, where $E G$ is a contractible space with a free $G$-action. Then one defines the Borel equivariant $h$-cohomology as $h_{G}(Y):=h\left(Y_{G}\right)$. This is not a new cohomology theory, but rather the nonequivariant theory applied to the Borel construction, a functor from $G$-spaces to spaces. That functor generalizes to orbifolds which are not necessarily global quotients - the functor is geometric realization - and so leads to a notion of "Borel cohomology" theories on orbifolds. But usually $h$ has other extensions to an equivariant theory. For example, the Atiyah-Segal geometric version of equivariant $K$-theory, defined in terms of equivariant vector bundles, is more delicate: Borel equivariant $K$-theory appears as a certain completion $[\mathbf{A S}]$. The Atiyah-Segal theory is extended to orbifolds, in fact to "local quotient groupoids", in [FHT].

We recalled at the beginning of $\S 1$ that the charges and fluxes associated to an abelian gauge field in a quantum gauge theory lie in generalized cohomology groups. When we pass to theories formulated on orbifolds we must additionally specify a flavor of equivariant cohomology to locate the charges and fluxes. For example, the Ramond-Ramond field in superstring theory has charges and fluxes in $K$-theory. In the corresponding orbifold theory they are in Atiyah-Segal equivariant $K$-theory. This choice has consequences even locally, at the level of differential forms: it is consistent with extra Ramond-Ramond fields in twisted sectors. We hope to elaborate in a future paper. Here we limit consideration to $B$-fields on orbifolds.

Let $M$ be a 26 -dimensional orbifold. We posit the following generalization of Supposition 1.1.

Supposition 3.1. For the oriented bosonic orbifold the flux of the $B$-field $\check{\beta}$ lies in the Borel cohomology $H^{3}(X ; \mathbb{Z})$.

Furthermore, there is a generalization of differential cohomology to orbifolds $[\mathbf{L U}, \mathbf{G}]$. So an immediate reformulation locates the $B$-field itself in orbifold differential cohomology (see (1.16)). Supposition 3.1 is implicit in the literature, for example in [Sh, GSW]. The $B$-field amplitude (2.1) is defined as before; the integration is still over a smooth manifold, the worldsheet $\Sigma$.

For the superstring case we also posit Borel cohomology for the $B$-field. Let $X$ be a 10-dimensional orbifold.

Proposal 3.2. For the superstring orbifold the flux of the B-field $\check{\beta}$ lies in the Borel cohomology $R^{-1}(X)$. 
We are not aware of any general equivariant version of generalized differential cohomology, much less a version for orbifolds. In [DFM2] we develop a geometric model of $\check{R}^{-1}(X)$ for a local quotient groupoid $X$ and locate the $B$-field there. The pullback to a worldsheet then lives in the differential $R$-theory as in the non-orbifold case, and the amplitude (2.1) is defined as before.

3.2. Orientifolds and $\boldsymbol{B}$-fields. The orientifold construction applies to both the bosonic string and the superstring. In its simplest incarnation the construction involves a pair $(Y, \sigma)$ of a smooth manifold $Y$ and an involution $\sigma: Y \rightarrow Y$. Fields on $Y$ have a definite transformation law under $\sigma$. For example, the metric is invariant whereas the 3 -form field strength $H$ of the $B$-field is anti-invariant: $\sigma^{*} H=-H$. We combine the orbifold and this simple orientifold by starting with a triple $(Y, \Gamma, v)$ consisting of a finite group $\Gamma$, a smooth $\Gamma$-manifold $Y$, and a surjective homomorphism $v: \Gamma \rightarrow$ $\mathbb{Z} / 2 \mathbb{Z}$. Then fields on $Y$ transform under $\Gamma$ : e.g., the 3 -form field strength of the $B$-field satisfies

$$
\gamma^{*} H=(-1)^{v(\gamma)} H, \quad \gamma \in \Gamma
$$

As before $\Gamma$ acts as a gauge symmetry and the physical points of spacetime lie in the quotient. Therefore, we arrive at a more general model in a geometric formulation.

DeFinition 3.4. The spacetime of an orientifold string model is an orbifold $X$ equipped with a double cover of orbifolds $\pi: X_{w} \rightarrow X$.

The equivalence class $w \in H^{1}(X ; \mathbb{Z} / 2 \mathbb{Z})$ of the double cover lies in the Borel cohomology of $X$. For the triple $(Y, \Gamma, v)$ the double cover is $\pi: Y / / \operatorname{ker} v \rightarrow$ $Y / / \Gamma$ with characteristic class in $H_{\Gamma}^{1}(Y ; \mathbb{Z} / 2 \mathbb{Z})$.

Definition 3.4 applies to both the bosonic string and the superstring. There is a particular special case of the orientifold construction which goes back to the early superstring theory literature.

Definition 3.5. The Type I superstring on a smooth 10-manifold $Y$ is the orientifold with spacetime $X=Y \times \mathrm{pt} / /(\mathbb{Z} / 2 \mathbb{Z})$, the orbifold quotient of the trivial involution on $Y$.

We next generalize Supposition 3.1 and Proposal 3.2 to bosonic and superstring orientifolds. First, recall that if $M$ is any space and $A \rightarrow M$ a fiber bundle of discrete abelian groups - a local system - then we can define twisted ordinary cohomology $H^{\bullet}(M ; A)$ with coefficients in $A$. In particular, if $M_{w} \rightarrow M$ is a double cover, then we form the associated bundle $A_{w} \rightarrow M$ of free abelian groups of rank one, defined by the action of $\{ \pm 1\}$ on $\mathbb{Z}$. We denote the associated twisted cohomology by $H^{w+\bullet}(M ; \mathbb{Z})$. It has a concrete manifestation in terms of cochain complexes: the deck transformation of 
the double cover $M_{w} \rightarrow M$ acts on the cochain complex $C^{\bullet}\left(M_{w} ; \mathbb{Z}\right)$, and $H^{w+\bullet}(M ; \mathbb{Z})$ is the cohomology of the anti-invariant subcomplex. If $M$ is a smooth manifold there is a corresponding twisted version $\check{H}^{w+\bullet}(M)$ of differential cohomology. We use the model of differential cohomology as a cochain complex of triples $(c, h, \omega)$, where $c \in C^{\bullet}\left(M_{w} ; \mathbb{Z}\right), \omega \in \Omega^{\bullet}\left(M_{w}\right)$, and $h \in C^{\bullet+1}\left(M_{w} ; \mathbb{R}\right)$ (see [DF, §6.3], [HS, §2.3]), and take the anti-invariant subcomplex.

Supposition 3.6. Let $X_{w} \rightarrow X$ be a double cover of 26-dimensional orbifolds and suppose $X$ is the spacetime of a bosonic orientifold. Then the flux of the $B$-field $\check{\beta}$ lies in the twisted Borel cohomology $H^{w+3}(X ; \mathbb{Z})$.

This appears in the literature using a different model of twisted degree three cohomology $[\mathbf{G S W}$. The equivalence class of the $B$-field lies in the twisted differential cohomology group $\check{H}^{w+3}(X)$, consistent with the transformation law (3.3).

The $B$-field quantization law for the superstring orientifold is expressed in terms of twisted $R$-cohomology. The following discussion applies to any cohomology theory $h$. Let $M_{w} \rightarrow M$ be a double cover of a space $M$ with deck transformation $\sigma$, and as after (1.3) let $\left\{h_{p}\right\}_{p \in \mathbb{Z}}$ denote a spectrum representing $h$-cohomology. Recall that $h^{p}(M)$ is the abelian group of homotopy classes of maps $M \rightarrow h_{p}$. Let $i_{p}: h_{p} \rightarrow h_{p}$ be a map which represents the additive inverse on cohomology classes, and we may assume $i_{p} \circ i_{p}=\mathrm{id}_{h_{p}}$. Define a $w$-twisted $h$-cocycle of degree $p$ on $M$ to be a pair $(c, \eta)$ of a map $c: M_{w} \rightarrow h_{p}$ and a homotopy $\eta$ from $\sigma^{*} c$ to $i_{p} c$. A homotopy of $w$-twisted $h$-cocycles is a $w$-twisted $h$-cocycle on $\Delta^{1} \times M$, where $\Delta^{1}$ is the 1 -simplex. Then $h^{w+p}(M)$ is defined as the group of homotopy classes of $w$-twisted $h$-cocycles of degree $p$. A small elaboration using triples as in (1.13) defines $w$-twisted $\breve{h}$-cohomology if $M$ is a smooth manifold. In [DFM2] we develop a differential-geometric model for $\check{R}^{w-1}(M)$.

Proposal 3.7. Let $X_{w} \rightarrow X$ be a double cover of 10-dimensional orbifolds and suppose $X$ is the spacetime of a superstring orientifold. Then the flux of the $B$-field $\check{\beta}$ lies in the twisted Borel cohomology $R^{w-1}(X)$.

REMARK 3.8. There is an important restriction on the $B$-field flux which we will derive in $\S 6$. Namely, a superstring orientifold spacetime $X$ carries a suitably twisted spin structure defined in terms of the $B$-field, and its existence leads to the constraints (6.9), (6.10).

3.3. Universal $\boldsymbol{B}$-fields on orientifolds. Let $B \mathbb{Z} / 2 \mathbb{Z}=\mathrm{pt} / /(\mathbb{Z} / 2 \mathbb{Z})$ and $\pi_{0}$ : pt $\rightarrow B \mathbb{Z} / 2 \mathbb{Z}$ the universal double cover, which we denote $w_{0}$. The geometric realization of $B \mathbb{Z} / 2 \mathbb{Z}$ is $\mathbb{R P}^{\infty}$, so the Borel $R$-cohomology of $B \mathbb{Z} / 2 \mathbb{Z}$ is the $R$-cohomology of $\mathbb{R} \mathbb{P}^{\infty}$. For orientifolds there are universal $B$-fields pulled back from the classifying map $X \rightarrow B \mathbb{Z} / 2 \mathbb{Z}$ of the orientifold 
double cover $X_{w} \rightarrow X$. For the bosonic orientifold we first apply the exact sequence analogous to (1.18),

$$
0 \longrightarrow H^{w+2}(M ; \mathbb{R} / \mathbb{Z}) \longrightarrow \check{H}^{w+3}(M) \longrightarrow \Omega_{\mathbb{Z}}^{w+3}(M) \longrightarrow 0,
$$

to $M=B \mathbb{Z} / 2 \mathbb{Z}$ and deduce $\check{H}^{w_{0}+3}(B \mathbb{Z} / 2 \mathbb{Z}) \cong H^{w_{0}+2}(B \mathbb{Z} / 2 \mathbb{Z} ; \mathbb{R} / \mathbb{Z})$. Now the twisted chain complex of the geometric realization $\mathbb{R P}^{\infty}$, starting in degree zero, is

$$
\mathbb{Z} \stackrel{2}{\longleftarrow} \mathbb{Z} \stackrel{0}{\longleftarrow} \mathbb{Z} \stackrel{2}{\longleftarrow} \mathbb{Z} \stackrel{0}{\longleftarrow} \mathbb{Z} \longleftarrow \cdots
$$

Apply $\operatorname{Hom}(-, \mathbb{R} / \mathbb{Z})$ to compute

$$
\check{H}^{w_{0}+3}(B \mathbb{Z} / 2 \mathbb{Z} ; \mathbb{Z}) \cong H^{w_{0}+2}(B \mathbb{Z} / 2 \mathbb{Z} ; \mathbb{R} / \mathbb{Z}) \cong \mathbb{Z} / 2 \mathbb{Z}
$$

This is the universal group of $B$-fields on bosonic orientifolds.

REMARK 3.12. The Bockstein map $H^{w_{0}+2}(B \mathbb{Z} / 2 \mathbb{Z} ; \mathbb{R} / \mathbb{Z}) \rightarrow H^{w_{0}+3}$ $(B \mathbb{Z} / 2 \mathbb{Z} ; \mathbb{Z})$ is an isomorphism, as follows easily from the long exact sequence associated to $\mathbb{Z} \rightarrow \mathbb{R} \rightarrow \mathbb{R} / \mathbb{Z}$. This is also obvious from the geometric picture of differential cohomology given around (1.14) since in this case $\check{H}^{w_{0}+3}(B \mathbb{Z} / 2 \mathbb{Z})$ is finite, hence equal to its group of components $H^{w_{0}+3}$ $(B \mathbb{Z} / 2 \mathbb{Z} ; \mathbb{Z})$.

For superstring orientifolds we also have a finite group of universal twistings.

THEOREM 3.13. The group $\check{R}^{w_{0}-1}(B \mathbb{Z} / 2 \mathbb{Z}) \cong R^{w_{0}-2}(B \mathbb{Z} / 2 \mathbb{Z} ; \mathbb{R} / \mathbb{Z}) \cong$ $R^{w_{0}-1}(B \mathbb{Z} / 2 \mathbb{Z} ; \mathbb{Z})$ is cyclic of order 8 . For any generator $\check{\theta}$ we can identify $4 \check{\theta}$ with the nonzero element in (3.11). Furthermore, the pullback of $\check{\theta}$ under $\pi_{0}: \mathrm{pt} \rightarrow B \mathbb{Z} / 2 \mathbb{Z}$ is $\check{\eta}$.

Recall that $\check{\eta}$ is the nonzero class in (1.20). In [DFM3] we interpret $R^{w_{0}-1}$ $(B \mathbb{Z} / 2 \mathbb{Z} ; \mathbb{Z})$ as a group of universal twistings of $K O$-theory (modulo Bott periodicity), which may be identified with the super Brouwer group [Wa, p. 195], [De, Proposition 3.6].

Proof. All cohomology groups in this proof have $\mathbb{Z}$ coefficients. We first show

$$
R^{w_{0}-1}(B \mathbb{Z} / 2 \mathbb{Z}):=R^{w-1}\left(\mathbb{R} \mathbb{P}^{\infty}\right) \cong R^{w-1}\left(\mathbb{R P}^{4}\right) \cong k o^{w-1}\left(\mathbb{R P}^{4}\right),
$$

where ' $w$ ' denotes the nontrivial double cover of projective space. The first equality is the definition of (twisted) Borel cohomology. The second group is computed as the space of sections of a twisted bundle of spectra over $\mathbb{R P}^{\infty}$ whose fiber is $R_{-1}$; see [ABGHR, MS]. The second isomorphism follows from elementary obstruction theory since $R_{-1}$ has vanishing homotopy groups above degree 3 ; see (1.5). Finally, the (-1)-space of the $k o$-spectrum 
and $R_{-1}$ have the same 5 -skeleton, which justifies the final isomorphism in (3.14).

Write $k o^{w-1}\left(\mathbb{R P}^{4}\right) \cong k o_{\mathbb{Z} / 2 \mathbb{Z}}^{w_{0}-1}\left(S^{4}\right)$. Here we use the Atiyah-Segal equivariant $k o$-theory for the antipodal action on the sphere; the equivariant double cover $w_{0}$ is pulled back from a point. Next, we claim

$$
k o_{\mathbb{Z} / 2 \mathbb{Z}}^{w_{0}-1}(\mathrm{pt}) \cong k o^{0}(\mathrm{pt}) .
$$

For in the Atiyah-Bott-Shapiro (ABS) model with Clifford algebras [ABS], the left hand side is the $K$-group of a category of $\mathbb{Z} / 2 \mathbb{Z}$-graded real modules for the $\mathbb{Z} / 2 \mathbb{Z}$-graded algebra $A$ generated by odd elements $\gamma, \alpha$ with $\gamma^{2}=-1, \alpha^{2}=1$, and $\alpha \gamma=-\gamma \alpha$. (That the generator $\alpha$ of $\mathbb{Z} / 2 \mathbb{Z}$ is odd reflects the twisting $w_{0}$; the Clifford generator $\gamma$ is always odd.) But $A$ is isomorphic to the $\mathbb{Z} / 2 \mathbb{Z}$-graded matrix algebra $\operatorname{End}\left(\mathbb{R}^{1 \mid 1}\right)$, and so the category of $A$-modules is Morita equivalent to the category of $\mathbb{Z} / 2 \mathbb{Z}$-graded real vector spaces. Let $^{9} \xi^{-1}$ denote the element in $k o_{\mathbb{Z} / 2 \mathbb{Z}}^{w_{0}-1}(\mathrm{pt})$ which corresponds to $1 \in k o^{0}(\mathrm{pt})$ under the isomorphism (3.15). In the ABS model $\xi^{-1}$ is represented by

$$
\xi^{-1}: \quad \mathbb{R}^{1 \mid 1} \text { with } \gamma=\left(\begin{array}{cc}
0 & -1 \\
1 & 0
\end{array}\right), \quad \alpha=\left(\begin{array}{ll}
0 & 1 \\
1 & 0
\end{array}\right) .
$$

Then multiplication by $\xi^{-1}$ induces an isomorphism $\widetilde{k o_{\mathbb{Z} / 2 \mathbb{Z}}^{0}}\left(S^{4}\right) \cong k o_{\mathbb{Z} / 2 \mathbb{Z}}^{w_{0}-1}$ $\left(S^{4}\right)$, where the tilde denotes reduced ko-theory. Now $\widetilde{k o_{\mathbb{Z} / 2 \mathbb{Z}}^{0}}\left(S^{4}\right) \cong \widetilde{k o}{ }^{0}$ $\left(\mathbb{R} P^{4}\right)$ and $\widetilde{k o}{ }^{0}\left(\mathbb{R P}^{4}\right)$ is cyclic of order 8 generated by $H-1$, where $H \rightarrow \mathbb{R P} \mathbb{P}^{4}$ is the nontrivial (Hopf) real line bundle: the order of $\widetilde{k o}^{0}\left(\mathbb{R P}^{4}\right)$ is bounded by 8 by the Atiyah-Hirzebruch spectral sequence, and because $w_{4}(4(H-$ 1)) $\neq 0$ we conclude $4(H-1) \neq 0$.

The assertion about $4 \check{\theta}$ follows from the twisted version of the exact sequence (1.6) on $B \mathbb{Z} / 2 \mathbb{Z}$ : the kernel group $H^{w_{0}+3}(B \mathbb{Z} / 2 \mathbb{Z} ; \mathbb{Z})$ is $(3.11)$. To prove the last statement we observe that the argument in the previous paragraph identifies the generator of $k o_{\mathbb{Z} / 2 \mathbb{Z}}^{w_{0}-1}\left(S^{4}\right)$ as the pullback of $\xi^{-1}$ under the $\mathbb{Z} / 2 \mathbb{Z}$-equivariant map $h: S^{4} \rightarrow$ pt. Let $i$ : pt $\hookrightarrow S^{4}$ be the (nonequivariant) inclusion of a point. Then $\pi_{0}^{*}(\check{\theta})$ is the image of $h^{*} \xi^{-1}$ under the composition $k o_{\mathbb{Z} / 2 \mathbb{Z}}^{w_{0}-1}\left(S^{4}\right) \rightarrow k o^{-1}\left(S^{4}\right) \stackrel{i^{*}}{\rightarrow} k o^{-1}(\mathrm{pt})$, which is evidently the image of $\xi^{-1}$ under $k o_{\mathbb{Z} / 2 \mathbb{Z}}^{w_{0}-1}(\mathrm{pt}) \rightarrow k o^{-1}(\mathrm{pt})$. (We choose orientations of pt and $S^{4}$ to trivialize the pullback of $w_{0}$ under $\pi_{0}$.) Finally, in the ABS model this pullback simply drops the action of $\alpha$, and what remains of (3.16) is the generator $\eta$ of $k o^{-1}(\mathrm{pt}) \cong \mathbb{Z} / 2 \mathbb{Z}$.

\footnotetext{
${ }^{9}$ We reserve the notation ' $\xi$ ' for the inverse class in twisted periodic $K O$-theory. It is the $K O$-Euler class of the real line with involution -1 , viewed as an equivariant line bundle over a point. It has many beautiful properties, some of which we exploit in [DFM3].
} 


\section{The $B$-field amplitude for orientifolds}

A worldsheet in an orientifold string theory has several fields [DFM1, Definition 5]. For the bosonic case they all appear in Definition 4.1; for the superstring there are additional fields articulated in Definition 4.8 and Definition 5.1.

4.1. Bosonic orientifold worldsheets. As a preliminary recall that a smooth $n$-manifold $M$ has a canonical orientation double cover $\hat{\pi}: \widehat{M} \rightarrow M$ defined as the quotient $\widehat{M}:=\mathcal{B}(M) / G L_{n}^{+} \mathbb{R}$, where $G L_{n}^{+} \mathbb{R}$ is the group of orientation-preserving automorphisms of $\mathbb{R}^{n}$. The manifold $\widehat{M}$ is canonically oriented. It is natural to denote the double cover $\hat{\pi}: \widehat{M} \rightarrow M$ as ' $w_{1}(M)$ '.

Definition 4.1. Let $\pi: X_{w} \rightarrow X$ be the spacetime of an orientifold string theory. An orientifold worldsheet is a triple $(\Sigma, \phi, \tilde{\phi})$ consisting of a compact 2-manifold $\Sigma$, a smooth map $\phi: \Sigma \rightarrow X$, and an equivariant lift $\tilde{\phi}: \widehat{\Sigma} \rightarrow X_{w}$ of $\phi$ to the orientation double cover of $\Sigma$.

In theories with open strings $\Sigma$ may have nonempty boundary. The surface $\Sigma$ is not oriented and need not be orientable. In fact, the existence of the equivariant lift implies a constraint involving its first Stiefel-Whitney class:

$$
\phi^{*} w=w_{1}(\Sigma)
$$

the equivariant lift $\tilde{\phi}$ is an isomorphism of the double covers in (4.2). ${ }^{10}$ For an orientifold spacetime defined by a triple $(Y, \Gamma, v)$ as above, Definition 4.1 unpacks to a principal $\Gamma$-bundle $P \rightarrow \Sigma$, an orientation on $P$, and a $\Gamma$-equivariant map $P \rightarrow Y$. There is a constraint: if $v(\gamma)=0$, then the action of $\gamma$ on $P$ preserves the orientation; if $v(\gamma)=1$, then $\gamma$ reverses the orientation. There is an obvious notion of equivalence of triples $(\Sigma, \phi, \tilde{\phi})$, and the collection of such triples forms a groupoid presentation of an infinite dimensional orbifold.

Remark 4.3. Definition 4.1 applied to a single string clarifies the nature of twisted sectors in orientifold theories. Namely, if $\phi: S^{1} \rightarrow X$ is a string, then the constraint implies that $\phi^{*} w=0$, since the circle is orientable. Thus $\phi$ lifts to the double cover $X_{w}$. Put differently, the homotopy class of $\phi$ does not detect a nontrivial double cover, so does not sense the orientifold. Now the "twisting" in a twisted sector for a global quotient orbifold $X=Y / / \Gamma$ measures the extent to which a string $S^{1} \rightarrow X$ fails to lift to a string $S^{1} \rightarrow Y$. So if $X=Y / / \Gamma$ is a global quotient with $v: \Gamma \rightarrow \mathbb{Z} / 2 \mathbb{Z}$ specifying the orientifold, then $\phi$ lifts to $X_{w}=Y / / \operatorname{ker} v$ and the twisted sectors are labeled by conjugacy classes in ker $v$. In case $X_{w}=Y$ is a smooth manifold and $X$ the orbifold quotient by an involution, then any string $\phi: S^{1} \rightarrow X$

\footnotetext{
${ }^{10}$ In our ambiguous notation ' $w$ ' and ' $w_{1}(M)$ ' denote both a double cover and its equivalence class.
} 
lifts to a loop $S^{1} \rightarrow Y$. Hence there are no twisted sectors in a "pure" orientifold.

4.2. $B$-field amplitudes for bosonic orientifolds. Recall that if $M$ is a smooth compact $n$-manifold then integration of differential forms

$$
\int_{M, \mathfrak{o}}: \Omega^{n}(M) \longrightarrow \mathbb{R}
$$

is only defined after choosing an orientation $\mathfrak{o}$. Absent an orientation one may integrate densities, which in our current notation are $w_{1}$-twisted differential forms: forms on the orientation double cover $\widehat{M}$ which are odd under the deck transformation. Integration of densities is a homomorphism

$$
\int_{M}: \Omega^{w_{1}(M)+n}(M) \longrightarrow \mathbb{R}
$$

which lifts to integration in twisted differential cohomology:

$$
\int_{M}: \check{H}^{w_{1}(M)+n+1}(M) \longrightarrow \check{H}^{1}(\mathrm{pt}) \cong \mathbb{R} / \mathbb{Z} .
$$

To define (4.6) one may follow [HS, §3.4] working in the model with smooth singular cochains.

That understood, the definition of the $B$-field amplitude (2.1) for bosonic orientifolds is straightforward. Let $\breve{\beta}$ be a bosonic orientifold $B$-field as in Supposition 3.6; its equivalence class is $[\check{\beta}] \in \check{H}^{w+3}(X)$. Then for an orientifold worldsheet as in Definition 4.1 the isomorphism (4.2) (defined by $\tilde{\phi}$ in Definition 4.1) places the pullback $\phi^{*}[\check{\beta}]$ in the group $\check{H}^{w_{1}(\Sigma)+3}(\Sigma)$. The $B$-field amplitude is then computed using a twisted integration (4.6) in place of (2.2). This bosonic orientifold $B$-field amplitude is described using a particular model for $\check{H}^{w+3}(X)$ in [GSW].

The universal $B$-field amplitude is easy to compute.

Proposition 4.7. Let $\check{\beta}$ be the nonzero universal B-field in (3.11). Then for any bosonic orientifold worldsheet the B-field amplitude (2.1) is $(-1)^{\operatorname{Euler}(\Sigma)}$, where $\operatorname{Euler}(\Sigma)$ is the Euler number of the closed surface $\Sigma$.

Proof. If $\phi: \Sigma \rightarrow X$ is the worldsheet map, then we can identify $\phi^{*}[\check{\beta}] \in$ $H^{w_{1}(\Sigma)+2}(\Sigma ; \mathbb{R} / \mathbb{Z})$ as the pullback of $x^{2} \in H^{2}\left(\mathbb{R P} \mathbb{P}^{\infty} ; \mathbb{Z} / 2 \mathbb{Z}\right)$ via the map $w_{1}: \Sigma \rightarrow \mathbb{R P}^{\infty}$ which classifies $w_{1}(\Sigma)$. The latter pulls back the generator $x \in H^{1}\left(\mathbb{R} \mathbb{P}^{\infty} ; \mathbb{Z} / 2 \mathbb{Z}\right)$ to $w_{1}(\Sigma)$, so $\phi^{*}[\check{\beta}]=w_{1}(\Sigma)^{2}$. Now $w_{1}(\Sigma)^{2}=w_{2}(\Sigma)$ since the difference of the two sides is the second Wu class, which vanishes on manifolds of dimension less than four. Finally, $w_{2}(\Sigma)$ is the mod 2 reduction of the Euler class (which in general lives in twisted integral cohomology). 
4.3. Spin structures on superstring orientifold worldsheets.

Turning to the worldsheet in a superstring orientifold theory we begin by specifying the appropriate notion of spin structure. We could not find this definition in the string theory literature, even for the Type I superstring.

DEFINITION 4.8. The topological data on a superstring orientifold worldsheet $\Sigma$ is a discrete field $\alpha$ which on each connected orientable open set $U \subset$ $\Sigma$ is a pair of spin structures which induce opposite orientations of $U$.

Definition 4.8 is identical to Definition 2.4 except for the omission of the global orientation. Although $\alpha$ is locally a pair of spin structures, there is no global spin structure on $\Sigma$. Rather, the local pair of spin structures with opposite underlying orientation glue to a global spin structure on the orientation double cover $\widehat{\Sigma}$. The global description is equivalent to the local Definition 4.8, and we use ' $\alpha$ ' to denote the spin structure on $\widehat{\Sigma}$ as well as the local field in Definition 4.8. Let $\hat{\sigma}$ denote the involution on $\widehat{\Sigma}$. If the spin structures are locally opposite consistent with gluing-more simply, if the pullback $\hat{\sigma}^{*} \alpha$ of the global spin structure on $\widehat{\Sigma}$ is the opposite $(-\alpha)$ - then a refinement to a $\mathrm{pin}^{-}$structure on $\Sigma$ may be possible, but is additional data.

REMARK 4.9. The oriented double cover $S^{2}$ of $\mathbb{R P}^{2}$ has a unique spin structure (up to $\cong$ ) compatible with the orientation. It refines to two inequivalent pin $^{-}$structures on $\mathbb{R} \mathbb{P}^{2}$. On the other hand, the oriented double cover $S^{1} \times S^{1}$ of the Klein bottle $K$ has 4 inequivalent spin structures compatible with the orientation. Two of them each refine in two inequivalent ways to give four inequivalent pin $^{-}$structures on the Klein bottle; the other two each refine in two inequivalent ways to give four inequivalent pin $^{+}$structures on the Klein bottle.

REMARK 4.10. It is important to emphasize that for general $\alpha$ there is no refinement to a $\mathrm{pin}^{-}$structure. (Indeed, if $\alpha$ refines to a $\mathrm{pin}^{-}$structure then the pullback to the orientation double cover is a spin structure which is invariant under the deck transformation.) This has important ramifications for the physics. Consider a connected open set $U \subset \Sigma$ with the topology of a cylinder. On $U$ there are four choices of a pair of spin structures: each spin structure can be either bounding or non-bounding when restricted to the circle. In the case where one spin structure bounds, and the other does not, it is impossible to refine $\alpha$ to a pin $^{-}$structure since the pullback of the pair to the oriented double cover of $U$ is not invariant under the deck transformation. From the physical viewpoint, it is clear from the Hamiltonian formulation of the string theory that this mixed choice of spin structures occurs for Feynman diagrams in which spacetime fermions propagate along an internal line corresponding to $U$. Conversely, restricting attention to only those $\alpha$ which do refine to a $\mathrm{pin}^{-}$structure misses all of the sectors of the worldsheet theory in which space-time fermions propagate along that channel. 
REMARK 4.11. Consider an orientifold theory in which the orientifold double cover $\pi: X_{w} \rightarrow X$ is trivial and trivialized. Then Definition 4.8 reduces to Definition 2.4. For the trivialization may be modeled as a section of $\pi$. Then for an orientifold worldsheet (Definition 4.1) $\phi: \Sigma \rightarrow X$ the equivariant lift $\tilde{\phi}$ identifies $\phi^{*}\left(X_{w} \rightarrow X\right) \cong(\widehat{\Sigma} \rightarrow \Sigma)$, and so the section of $\pi$ pulls back to a section of $\hat{\pi}: \widehat{\Sigma} \rightarrow \Sigma$. But the latter is precisely a global orientation $\mathfrak{o}$ of $\Sigma$.

4.4. $B$-field amplitudes for superstring orbifolds. To describe the $B$-field amplitude (2.1) for the superstring we need the analog of (4.6) in differential $R$-theory. A complete definition involves twistings of cohomology theories beyond twists by double covers (see the discussion preceding Proposal 3.7) and is deferred to [DFM2]. For now recall that $R$ is Spinoriented and there is a pushforward (2.6) on spin manifolds. More generally, the obstruction to a spin structure on an $n$-manifold $M$ determines a twisting $\tau^{R}(M)$ of $R$-theory, so too of differential $R$-theory, and a twisted pushforward

$$
\int_{M}: \check{R}^{\tau^{R}(M)-3}(M) \longrightarrow \check{R}^{-3}(\mathrm{pt}) \cong \mathbb{R} / \mathbb{Z}
$$

The twisting $\tau^{R}(M)$ includes the dimension of $M$, as well as the StiefelWhitney classes $w_{1}(M), w_{2}(M)$. A spin structure produces an isomorphism $n \rightarrow \tau^{R}(M)$ and so reduces the pushforward (4.12) to a pushforward on untwisted differential $R$-theory, as in (2.6).

Now suppose $\pi: X_{w} \rightarrow X$ is the orientifold double cover of a 10-dimensional superstring spacetime $X$ with $B$-field $\check{\beta}$. Given a worldsheet as in Definitions 4.1 and 4.8 the pullback $\phi^{*}[\check{\beta}]$ of the equivalence class of the $B$-field lies in $\check{R}^{w_{1}(\Sigma)-1}(\Sigma)$. It seems, then, that to push forward to a point using (4.12) we need an isomorphism $w_{1}(\Sigma) \rightarrow \tau^{R}(\Sigma)-2$ of twistings of $R$-theory. However, the local spin structures $\alpha$ on $\Sigma$-equivalently global spin structure on $\widehat{\Sigma}$ - do not give such an isomorphism. This puzzle stymied the authors for a long period. The resolution is that the $B$-field amplitude in general is not a number, but rather an element in a complex line:

\section{(4.13) The B-field amplitude for a superstring orientifold is anomalous. ${ }^{11}$}

There is one case in which there is an isomorphism $w_{1}(\Sigma) \rightarrow \tau^{R}(\Sigma)-$ 2 , namely when $\alpha$ is refined to a pin $^{-}$structure on $\Sigma$. Then the $B$-field amplitude may be defined as a number. Notice that on a pin $^{-}$worldsheet the two local spin structures $\alpha$ are opposites. The anomaly measures the extent to which that fails for general $\alpha$.

\footnotetext{
${ }^{11}$ We refer to a term in an (effective) action as anomalous if it takes values in a (noncanoncially trivialized) complex line rather than the complex numbers.
} 
REMARK 4.14. To illustrate, suppose that the superstring orientifold worldsheet $\Sigma$ is diffeomorphic to a 2-dimensional torus. Even though $\Sigma$ is orientable, the fields do not include an orientation. The field $\alpha$ is equivalent to a pair of spin structures $\alpha^{\prime}, \alpha^{\prime \prime}$ on $\Sigma$ with opposite underlying orientations. Up to isomorphism there are 4 choices for each of $\alpha^{\prime}, \alpha^{\prime \prime}$, so 16 possibilities in total. Of those 4 refine uniquely to pin $^{-}$structures on $\Sigma$. The $B$-field amplitudes for the remaining 12 are anomalous.

Recall from Theorem 2.9 that in the oriented case the universal $B$-field amplitude for the superstring computes the well-known $\mathbb{Z} / 2 \mathbb{Z}$-valued quadratic form on spin structures. We now investigate the analogous amplitude in the orientifold case for $\mathrm{pin}^{-}$worldsheets. Let $\Sigma$ be a closed 2 -manifold and $\mathcal{P}^{-}(\Sigma)$ the $H^{1}(\Sigma ; \mathbb{Z} / 2 \mathbb{Z})$-torsor of equivalence classes of pin $^{-}$structures. Let $\check{\theta}$ be a generator of the cyclic group $R^{w_{0}-2}(B \mathbb{Z} / 2 \mathbb{Z} ; \mathbb{R} / \mathbb{Z})$; see Theorem 3.13 . Now the orientation double cover determines a map $h: \Sigma \rightarrow B \mathbb{Z} / 2 \mathbb{Z}$ and so a class $h^{*} \check{\theta} \in R^{w_{1}(\Sigma)-2}(\Sigma ; \mathbb{R} / \mathbb{Z})$. Let $p: \Sigma \rightarrow$ pt. Then a pin ${ }^{-}$structure $\alpha^{-}$ on $\Sigma$ determines a pushforward map

$$
p_{*}^{\alpha^{-}}: R^{w_{1}(\Sigma)-2}(\Sigma ; \mathbb{R} / \mathbb{Z}) \longrightarrow R^{-4}(\mathrm{pt} ; \mathbb{R} / \mathbb{Z}) \cong \mathbb{R} / \mathbb{Z} .
$$

Define

$$
\begin{aligned}
& q^{-}: \mathcal{P}^{-}(\Sigma) \longrightarrow \mathbb{R} / \mathbb{Z} \\
& \alpha^{-} \longmapsto p_{*}^{\alpha^{-}}\left(h^{*} \check{\theta}\right)
\end{aligned}
$$

We can replace the $R$-cohomology groups in (4.15) with ko-groups or even periodic $K O$-groups.

THEOREM 4.17. The function $q^{-}$takes values in $\frac{1}{8} \mathbb{Z} / \mathbb{Z} \cong \mathbb{Z} / 8 \mathbb{Z}$, is a quadratic refinement of the intersection pairing, and its reduction modulo two is congruent to the Euler number Euler $(\Sigma)$.

Proof. The first statement follows since $8 \check{\theta}=0$. We must show that for $a_{1}, a_{2} \in H^{1}(\Sigma ; \mathbb{Z} / 2 \mathbb{Z})$,

$q^{-}\left(\alpha^{-}+a_{1}+a_{2}\right)-q^{-}\left(\alpha^{-}+a_{1}\right)-q^{-}\left(\alpha^{-}+a_{2}\right)+q^{-}\left(\alpha^{-}\right)=\frac{1}{2} a_{1} \cdot a_{2}, \quad \alpha^{-} \in \mathcal{P}^{-}(\Sigma)$.

The argument of $[\mathbf{A 1}$, p. 53] applies verbatim through Lemma (2.3), which we replace with the following assertion. Let $i$ : pt $\hookrightarrow \Sigma$ and $u=i_{*}\left(\eta^{2}\right) \in$ $k o^{0}(\Sigma ; \mathbb{Z})$; then

$$
p_{*}^{\alpha^{-}}\left(h^{*} \check{\theta} \cdot u\right)=1 / 2 .
$$

To prove this we note that $u$ is supported in a neighborhood of a point in $\Sigma$, so by excision we can compute the left side on a sphere $S^{2}$. Fix an orientation of $S^{2}$, which is a section of the orientation double cover $w_{1}(\Sigma)$. 
This lifts $h: S^{2} \rightarrow B \mathbb{Z} / 2 \mathbb{Z}$ to $\pi_{0}:$ pt $\rightarrow B \mathbb{Z} / 2 \mathbb{Z}$. Then since by Theorem 3.13 we have $\pi_{0}^{*} \check{\theta}=\check{\eta}$, we reduce $(4.19)$ to $p_{*}(\check{\eta} \cdot u)$, which by push-pull is $\check{\eta} \cdot \eta^{2}$. As in the proof of Theorem 2.9 this is nonzero.

The last statement follows from Proposition 4.7 since $4 \check{\theta}$ is the nonzero element of (3.11); see Theorem 3.13.

Recall [KT, §3] that the $\mathrm{pin}^{-}$bordism group $\Omega_{2}^{\mathrm{Pin}^{-}}$is cyclic of order eight and the Kervaire invariant is an isomorphism.

COROLlaRY 4.20. With an appropriate choice of generator $\check{\theta}$ in Theorem 3.13, the quadratic form (4.16) is the Kervaire invariant.

For oriented surfaces the $\mathbb{Z} / 2 \mathbb{Z}$-valued Kervaire invariant (2.7) has a wellknown $K O$-theoretic interpretation [A1]. Corollary 4.20 provides a similar $K O$-theoretic interpretation in the unoriented case.

Proof. The definition (4.16) of $q^{-}$is evidently a bordism invariant. The real projective plane $\mathbb{R P}^{2}$ has two pin ${ }^{-}$structures; either generates $\Omega_{2}^{\mathrm{Pin}^{-}}$. Since $\mathbb{R P}^{2}$ has odd Euler number, the value of $q^{-}$on either pin $^{-}$structure is a generator of $\mathbb{Z} / 8 \mathbb{Z}$. The four possible choices of $\check{\theta}$ in the definition of $q^{-}$ give the four generators of $\mathbb{Z} / 8 \mathbb{Z}$, so we can choose the one which matches the standard Kervaire invariant on $\mathbb{R P}^{2}$, hence on all pin ${ }^{-}$surfaces.

\section{Worldsheet fermions and spacetime spin structures}

A fermionic functional integral is, by definition, the pfaffian of a Dirac operator. It is naturally an element of a line, so in a family of bosonic fields a section of a line bundle over the parameter space $[\mathbf{F 1}$, Part 2]. For an orientifold superstring worldsheet the $B$-field amplitude is also anomalous (4.13). The main result of [DFM2] is that the product of these anomalies is trivializable, and furthermore the correct notion of spin structure on spacetime ( $\S 6)$ leads to a trivialization. In this section, after identifying the fermionic fields in the 2-dimensional worldsheet theory, we work out an analogous phenomenon in a familiar 1-dimensional theory: the "spinning particle". Namely, in Theorem 5.11 we identify the pfaffian line of the Dirac operator on a circle in terms of the frame bundle of spacetime, and show how a spin structure on spacetime leads to a trivialization.

5.1. Fermions on orientifold superstring worldsheets. This is the last in the triad of definitions (see Definitions 4.1 and 4.8) specifying the fields on an orientifold superstring worldsheet [DFM1, Definition 5].

Definition 5.1. An orientifold superstring worldsheet consists of $(\Sigma, \phi$, $\tilde{\phi}, \alpha)$ as in Definitions 4.1 and 4.8 together with a positive chirality spinor field $\psi$ on $\widehat{\Sigma}$ with coefficients in $\hat{\pi}^{*} \phi^{*} T X$ and a negative chirality spinor field $\chi$ on $\widehat{\Sigma}$ with coefficients in $T^{*} \widehat{\Sigma}$. 
The notion of chirality is defined by the canonical orientation on the orientation double cover $\widehat{\Sigma}$; the spinors use the spin structure $\alpha$. Both $\psi$ (the "matter fermion") and $\chi$ (the "gravitino") should be regarded as local fields on $\Sigma$, but the global description on $\widehat{\Sigma}$ is more transparent; the action is local on $\Sigma$. The crucial factor in the functional integral over $\psi, \chi$ for fixed $\phi$ and $\alpha$ is the pfaffian of a Dirac operator on $\widehat{\Sigma}$, which may be written

$$
\text { pfaff } D_{\widehat{\Sigma}, \alpha}\left(\hat{\pi}^{*} \phi^{*} T X-T \widehat{\Sigma}\right) .
$$

The pfaffian line bundle is local, so we can heuristically analyze it on a small contractible open set $U \subset \Sigma$. Now $\pi^{-1} U \subset \widehat{\Sigma}$ is the disjoint union of two oppositely oriented open sets diffeomorphic to $U$ with spin structures $\alpha^{\prime}, \alpha^{\prime \prime}$ refining the underlying orientations. The pfaffian (5.2) is anomalous on each component of $\pi^{-1} U$. If the spin structures $\alpha^{\prime}, \alpha^{\prime \prime}$ are opposite, then the product of the anomalies is trivializable; an isomorphism of $\alpha^{\prime}$ with the opposite of $\alpha^{\prime \prime}$ trivializes the anomaly. So we see that the anomaly measures the failure of $\alpha^{\prime}$ and $\alpha^{\prime \prime}$ to be opposites, just as for the $B$-field. ${ }^{12}$ (See the text leading to Remark 4.14.)

For the oriented superstring a global argument for the triviality of the pfaffian line bundle - the anomaly in the fermionic functional integral (5.2) is given in $[\mathbf{F W}, \S 4]$. In the non-orientifold case there is no anomaly in the $B$-field amplitude (see (2.6)). The argument in $[\mathbf{F W}]$ only proves the triviality; it does not provide a trivialization so does not determine a definition of (5.2) as a function. (This additional data is sometimes termed a 'setting of the quantum integrand'.) In fact, the superstring data does determine a trivialization: it is the spacetime spin structure which is critical. We explore this two-dimensional anomaly problem in [DFM2] and show that the trivialization varies under a change of spacetime spin structure.

REMARK 5.3. For an oriented superstring worldsheet (Definition 2.4), the dependence is as follows. Suppose $a \in H^{1}(X ; \mathbb{Z} / 2 \mathbb{Z})$ is a change of spacetime spin structure and $b=\alpha_{l}-\alpha_{r} \in H^{1}(\Sigma ; \mathbb{Z} / 2 \mathbb{Z})$ the difference of the two global worldsheet spin structures. Then the trivialization for a worldsheet $\phi: \Sigma \rightarrow X$ multiplies by

$$
(-1)^{\left\langle\phi^{*} a, b\right\rangle}
$$

where $\langle-,-\rangle$ is the $\mathbb{Z} / 2 \mathbb{Z}$-valued pairing on $H^{1}(\Sigma ; \mathbb{Z} / 2 \mathbb{Z})$. Combining this factor with (2.14) one sees that our formulation of the oriented superstring has the expected left-right symmetry. See (5.10) for a 1-dimensional analog of (5.4). Equation (5.4) is consistent with [AW].

5.2. A supersymmetric quantum mechanical theory. Here we illustrate the impact of the spacetime spin structure on the worldsheet pfaffian in a simpler quantum field theory: the 1-dimensional supersymmetric

\footnotetext{
${ }^{12}$ The anomaly also depends on the topology of $\phi^{*}(T X)$.
} 
quantum mechanical system whose partition function computes the index of the Dirac operator $[\mathbf{A g}, \mathbf{F W}, \mathbf{W} \mathbf{1}]$. In this theory spacetime $X$ is a Riemannian manifold of arbitrary dimension $n$. For the classical theory it does not have a spin structure or even an orientation. However, to simplify we assume that $X$ is oriented. The worldsheet of superstring theory is replaced by a 1-dimensional manifold $S$ with a map $\phi: S \rightarrow X$. The manifold $S$ is endowed with a single spin structure. The fermionic fields of Definition 5.1 are replaced by a single spinor field $\psi$ on $S$ with coefficients in $\phi^{*} T X$.

Consider $S=S^{1}$ with the nonbounding spin structure $\alpha$. The first step in computing the partition function is to compute the fermionic functional integral over $\psi$ for a fixed loop $\phi: S^{1} \rightarrow X$, which is the pfaffian

$$
\text { pfaff } D_{S^{1}, \alpha}\left(\phi^{*} T X\right) \text {. }
$$

As the Dirac operator on the circle is real, the square of its pfaffian line bundle is canonically trivial and so the square of (5.5) is a well-defined function. There is a standard regularization and the result (see $[\mathbf{A 3}]$, for example) is

$$
\left(\operatorname{pfaff} D_{S^{1}, \alpha}\left(\phi^{*} T X\right)\right)^{2}=\operatorname{det}(1-\operatorname{hol}(\phi)),
$$

where $\operatorname{hol}(\phi) \in S O_{n}$ is the holonomy, well-defined up to conjugacy. We may as well assume that $n$ is even, or else (5.6) vanishes identically. Now the function $g \mapsto \operatorname{det}(1-g)$ on $S O_{n}$ does not have a smooth square root. However, its lift to $\operatorname{Spin}_{n}$ does have a square root $f$, the difference of the characters of the half-spin representations:

$$
f(\tilde{g})=i^{n / 2}\left(\chi_{\Delta^{+}}(\tilde{g})-\chi_{\Delta^{-}}(\tilde{g})\right), \quad \tilde{g} \in \operatorname{Spin}_{n} .
$$

Hence given a spin structure on $X$ we can lift the holonomy function hol: $L X \rightarrow S O_{n}$ on the loop space of $X$ to a function $\widetilde{\text { hol }}: L X \rightarrow \operatorname{Spin}_{n}$, and so define (5.5) as

$$
\text { pfaff } D_{S^{1}, \alpha}\left(\phi^{*} T X\right):=f(\widetilde{\operatorname{hol}}(\phi)) .
$$

The right hand side of (5.8) manifestly uses the spin structure on spacetime $X$. Note that we can equally replace the function $f$ by its negative; the overall sign is not determined by this argument.

REMARK 5.9. If we change the spin structure on $X$ by a class $a \in$ $H^{1}(X ; \mathbb{Z} / 2 \mathbb{Z})$, then it follows immediately from (5.8) that the pfaffian multiplies by

$$
(-1)^{\phi^{*}(a)\left[S^{1}\right]} .
$$

The pfaffian is more naturally an element of a line and for the analogy with the 2-dimensional worldsheet theory it is more illuminating to analyze 
the pfaffian line Pfaff $D_{S^{1}, \alpha}\left(\phi^{*} T X\right)$ directly. (See $[\mathbf{F 2}, \S 3]$ for the definition of the pfaffian line of a Dirac operator.) Write $E \rightarrow S^{1}$ for the oriented vector bundle $\phi^{*} T X$. The Dirac operator $D_{S^{1}, \alpha}$ is the covariant derivative $\nabla$ acting on sections of $E \rightarrow S^{1}$. It is real and skew-adjoint, so its pfaffian line is real. Replace a real line $L$ by the $\mathbb{Z} / 2 \mathbb{Z}$-torsor $\pi_{0}(L \backslash\{0\})$, so obtain the pfaffian torsor Pfaff $\nabla$. Let $\mathcal{B}_{S O}(E) \rightarrow S^{1}$ denote the bundle of oriented orthonormal frames of $E$. It is trivializable since $S O_{n}$ is connected. The space of sections $\Gamma$ has two components and is naturally a torsor for $\pi_{1}\left(S O_{n}\right) \cong$ $\mathbb{Z} / 2 \mathbb{Z}$. Furthermore, a spin structure $\mathcal{B}_{\text {Spin }} \rightarrow \mathcal{B}_{S O}(E) \rightarrow S^{1}$ trivializes the torsor $\pi_{0} \Gamma$ : there is a distinguished component of sections which lift to $\mathcal{B}_{\text {Spin }}$.

THEOREM 5.11. There is a canonical isomorphism Pfaff $\nabla \cong \pi_{0} \Gamma$. Therefore, a spin structure on $E$ determines a trivialization of Pfaff $\nabla$.

Suppose $Z$ is any manifold and $E \rightarrow Z \times S^{1}$ an oriented bundle with covariant derivative. Then the Pfaffian torsors vary smoothly in $z \in Z$ so form a double cover of $Z$. Its characteristic class may be computed from the Atiyah-Singer index theorem as the slant product $w_{2}(E) /\left[S^{1}\right]$; see $[\mathbf{F W}$, (5.22)]. Theorem 5.11 is a "categorification" of this topological result-an isomorphism of line bundles rather than simply an equality of their isomorphism classes - necessary in order to discuss trivializations. We remark that more sophisticated categorifications of the Atiyah-Singer index theorem are needed for anomaly problems in higher dimensions, such as [DFM2]; see $[\mathbf{B u}]$ for a recent result in dimension two.

Proof. Fix a Riemannian metric on $S^{1}$ of total length 1 . The covariant derivative of a framing $e \in \Gamma$ is a function $\nabla(e): S^{1} \rightarrow \mathfrak{s o}_{n}$. Using parallel transport choose $e$ so that $\nabla(e)$ is a constant skew-symmetric matrix $A$ whose eigenvalues $a \sqrt{-1}$ satisfy $-\pi<a \leq \pi$. Note that $\exp (A)$ is the holonomy of $\nabla$. The framing $e$ is determined up to a constant element of $S O_{n}$. In particular, the span $W$ of the basis vectors of $e$ in the space $\mathcal{H}$ of sections of $E \rightarrow S^{1}$ is independent of this choice. It is easy to see that $\nabla$ is invertible on the orthogonal complement $W^{\perp}$ to $W$ in $\mathcal{H}$ relative to the $L^{2}$ metric. So Pfaff $\nabla$ is canonically the determinant line Det $W^{*}$ of the finite dimensional vector space $W^{*}$, and the associated $\mathbb{Z} / 2 \mathbb{Z}$-torsor is canonically the $\mathbb{Z} / 2 \mathbb{Z}$ torsor $\mathcal{T}$ of orientation classes of bases of $W$. But a basis of $W$ is an element of $\Gamma$, so $\mathcal{T}$ is canonically $\pi_{0} \Gamma$, as claimed.

REMARK 5.12. Formula (5.10) for the change of trivialization as a function of the change of spin structure on $E$ follows immediately: $E \rightarrow S^{1}$ has two spin structures and they determine two different points of $\pi_{0} \Gamma$.

\section{The twisted spin structure on a superstring orientifold spacetime}

The spacetime $X$ of an oriented superstring theory has a spin structure. There is a modification for orientifolds in superstring theory: the notion 
of spin structure is twisted by both the orientifold double cover $\pi: X_{w} \rightarrow$ $X$ and the $B$-field. In this section we describe this twisted notion of spin structure in concrete differential-geometric terms.

Recall quite generally that if $\rho: G \rightarrow G^{\prime}$ is a homomorphism of Lie groups and $P \rightarrow M$ a principal $G$-bundle over a space $M$, then there is an associated principal $G^{\prime}$-bundle $\rho(P) \rightarrow M$, defined by the "mixing construction" $\rho(P)=P \times{ }_{G} G^{\prime}$. Conversely, if $Q \rightarrow M$ is a principal $G^{\prime}$-bundle, then a reduction to $G$ along $\rho$ is a pair $(P, \varphi)$ consisting of a principal $G$-bundle $P \rightarrow M$ and an isomorphism $\varphi: \rho(P) \rightarrow Q$. If $M^{n}$ is a smooth manifold and $\rho: G \rightarrow G L_{n} \mathbb{R}$, then a reduction of the $G L_{n} \mathbb{R}$ frame bundle $\mathcal{B}(M)$ to $G$ along $\rho$ is called a $G$-structure on $M$. We defined orientations in these terms in $\S 4.1$ and spin structures in these terms in $\S 2.1$; for convenience we used a metric and so a homomorphism (2.3) into the orthogonal group. A principal $G$-bundle is classified by a map ${ }^{13} M \rightarrow B G$ whose homotopy class is an invariant of $P \rightarrow M$. The topological classification of reductions along $\rho: G \rightarrow G^{\prime}$ may be analyzed as a lifting problem:

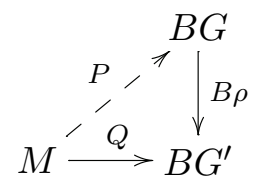

Two particular cases are of interest here: (i) $\rho$ is the inclusion of an index two subgroup, in which case $B \rho: B G \rightarrow B G^{\prime}$ is a double cover and the obstruction to (6.1) lies in $H^{1}(M ; \mathbb{Z} / 2 \mathbb{Z}$ ); and (ii) $\rho$ is a surjective double cover, in which case $B \rho: B G \rightarrow B G^{\prime}$ is a principal $K(\mathbb{Z} / 2 \mathbb{Z}, 1)$-bundle ${ }^{14}$ and the obstruction to $(6.1)$ lies in $H^{2}(M ; \mathbb{Z} / 2 \mathbb{Z})$.

The spin group (2.3) is a double cover of an index two subgroup of $O_{n}$. We now define groups $G_{0}, G_{1}$ which bear the same relation to $\widetilde{O}_{n}:=O_{n} \times$ $\mathbb{Z} / 2 \mathbb{Z} \times \mathbb{Z} / 2 \mathbb{Z}$ via homomorphisms

$$
\rho_{i}: G_{i} \longrightarrow \widetilde{O}_{n}, \quad i=1,2
$$

which factor through an index two subgroup $G_{i}^{\prime} \subset \widetilde{O}_{n}$. First, let $D_{4} \rightarrow$ $\mathbb{Z} / 2 \mathbb{Z} \times \mathbb{Z} / 2 \mathbb{Z}$ be the dihedral double cover in which the generators of the $\mathbb{Z} / 2 \mathbb{Z}$ factors lift to anticommuting elements of order two. Define $G_{0}, G_{0}^{\prime}$ as the first two groups in

$$
\rho_{0}:\left(\operatorname{Spin}_{n} \times D_{4}\right) /\{ \pm 1\} \longrightarrow S O_{n} \times \mathbb{Z} / 2 \mathbb{Z} \times \mathbb{Z} / 2 \mathbb{Z} \longrightarrow \widetilde{O}_{n},
$$

\footnotetext{
${ }^{13}$ More precisely, a classifying map for $P \rightarrow M$ is a $G$-equivariant map $P \rightarrow E G$ for $E G \rightarrow B G$ a universal $G$-bundle.

${ }^{14} K(\mathbb{Z} / 2 \mathbb{Z}, 1)$ is an Eilenberg-MacLane space; a topological group model is the group of projective linear transformations of an infinite dimensional real Hilbert space.
} 
where $-1 \in\{ \pm 1\}$ is the product of the central elements of $\operatorname{Spin}_{n}$ and $D_{4}$. For $G_{1}$ we first define the surjective homomorphism

$$
\begin{aligned}
\widetilde{O}_{n} & \longrightarrow \mathbb{Z} / 2 \mathbb{Z} \\
(g, a, b) & \longmapsto c+a, \quad \operatorname{det}(g)=(-1)^{c},
\end{aligned}
$$

and let $G_{1}^{\prime}$ be the kernel. Then $G_{1}$ is the inverse image of $G_{1}^{\prime}$ under

$$
\left(\operatorname{Pin}_{n}^{-} \times D_{4}\right) /\{ \pm 1\} \longrightarrow \widetilde{O}_{n} .
$$

Suppose $X$ is a superstring spacetime - a 10-dimensional orbifold - and $X_{w} \rightarrow X$ an orientifold double cover. Proposal 3.7 implies that a $B$-field $\check{\beta}$ is a geometric object whose equivalence class $[\check{\beta}]$ lies in $\check{R}^{w-1}(X)$. As in (1.17) there are topological invariants $t(\check{\beta}): \pi_{0} X \rightarrow \mathbb{Z} / 2 \mathbb{Z}$ and a double cover $X_{a(\check{\beta})} \rightarrow X$.

Definition 6.6. Let $X_{w} \rightarrow X$ be the orientifold double cover of a Riemannian orbifold $X$ which represents a superstring spacetime. Let $\breve{\beta}$ be a $B$-field on $X$. Then a twisted spin structure is a reduction of the principal $\widetilde{O}_{10}$-bundle

$$
\mathcal{B}_{O}(X) \times_{X} X_{w} \times_{X} X_{a(\breve{\beta})} \rightarrow X
$$

along $\rho: G_{i} \rightarrow \widetilde{O}_{10}$, where $i \in \mathbb{Z} / 2 \mathbb{Z}$ is chosen on each component of $X$ according to the value of $t(\check{\beta})$.

Definition 2.11 expresses the two types in more familiar terms as Type IIB for $t(\check{\beta})=0$ and Type IIA for $t(\breve{\beta})=1$. Typically spacetime is connected and only one of these occurs.

Equivalence classes of twisted spin structures, if they exist, form a torsor for $H^{0}(X ; \mathbb{Z} / 2 \mathbb{Z}) \times H^{1}(X ; \mathbb{Z} / 2 \mathbb{Z})$. The existence is settled by the following.

Proposition 6.8. Let $X_{w} \rightarrow X$ and $\check{\beta}$ be as in Definition 6.6. Then a twisted spin structure exists if and only if

$$
\begin{aligned}
& w_{1}(X)=t(\check{\beta}) w \\
& w_{2}(X)=a(\check{\beta}) w+t(\check{\beta}) w^{2}
\end{aligned}
$$

These equations live in the Borel cohomology of the orbifold $X$.

Proof. Equation (6.9) is the condition to reduce the structure group of (6.7) along the inclusion $G_{i}^{\prime} \hookrightarrow \widetilde{O}_{10}$. For $G_{0}^{\prime}=S O_{10} \times \mathbb{Z} / 2 \mathbb{Z} \times \mathbb{Z} / 2 \mathbb{Z}$ it is the condition $w_{1}(X)=0$ for an orientation. For $t(\check{\beta})=1$ the homomorphism (6.4) induces a map $B \widetilde{O}_{10} \rightarrow B \mathbb{Z} / 2 \mathbb{Z}$ which pulls the generator of $H^{1}(B \mathbb{Z} / 2 \mathbb{Z} ; \mathbb{Z} / 2 \mathbb{Z})$ back to $w_{1}+x$, where $H^{1}\left(B \widetilde{O}_{10} ; \mathbb{Z} / 2 \mathbb{Z}\right)=H^{1}\left(B O_{10}\right.$; $\mathbb{Z} / 2 \mathbb{Z}) \times H^{1}(B \mathbb{Z} / 2 \mathbb{Z} ; \mathbb{Z} / 2 \mathbb{Z}) \times H^{1}(B \mathbb{Z} / 2 \mathbb{Z} ; \mathbb{Z} / 2 \mathbb{Z})$ has generators $w_{1}, x, y$. Then (6.9) follows by pullback along the classifying map of (6.7). 
For (6.10) we first observe that the double cover $D_{4} \rightarrow \mathbb{Z} / 2 \mathbb{Z} \times \mathbb{Z} / 2 \mathbb{Z}$ is classified by $x y \in H^{2}(B \mathbb{Z} / 2 \mathbb{Z} \times B \mathbb{Z} / 2 \mathbb{Z} ; \mathbb{Z} / 2 \mathbb{Z})$. Then the first homomorphism in (6.3) induces a principal $K(\mathbb{Z} / 2 \mathbb{Z}, 1)$-bundle $B G_{0} \rightarrow B G_{0}^{\prime}$ classified by $w_{2}+x y$, from which (6.10) follows on components with $t(\check{\beta})=$ 0 . For components with $t(\check{\beta})=1$ we first recall [KT, Lemma 1.3] that the universal $K(\mathbb{Z} / 2 \mathbb{Z}, 1)$-bundle $B \operatorname{Pin}_{10}^{-} \rightarrow B O_{10}$ is classified by $w_{1}^{2}+$ $w_{2} \in H^{2}\left(B O_{10} ; \mathbb{Z} / 2 \mathbb{Z}\right)$. Then the definition (6.5) of $G_{1}$ shows that $B G_{1} \rightarrow$ $B G_{1}^{\prime}$ is classified by $w_{1}^{2}+w_{2}+x y$; equation (6.10) now follows from this and (6.9).

REMARK 6.11. The occurrence of $D_{4}$ in our definition of a twisted spin structure is closely related to the $D_{4}$ symmetry group ${ }^{15}$ which appears in Hamiltonian treatments of orientifolds in the physics literature. We hope to elaborate on this elsewhere.

\section{References}

[A1] Michael F. Atiyah, Riemann surfaces and spin structures, Ann. Sci. École Norm. Sup. (4) 4 (1971), 47-62.

[A2] M. F. Atiyah, K-Theory, second ed., Advanced Book Classics, AddisonWesley, Redwood City, CA, 1989.

[A3] Circular symmetry and stationary-phase approximation, Astérisque (1985), no. 131, 43-59. Colloquium in honor of Laurent Schwartz, Vol. 1 (Palaiseau, 1983).

[ABGHR] M. Ando, A. J. Blumberg, D. J. Gepner, M. J. Hopkins, and C. Rezk, Units of ring spectra and Thom spectra, arXiv:0810.4535.

[ABS] M. F. Atiyah, R. Bott, and A. Shapiro, Clifford modules, Topology 3 (1964), no. suppl. 1, 3-38.

[Ag] Luis Alvarez-Gaume, Supersymmetry and the Atiyah-Singer index theorem, Commun. Math. Phys. 90 (1983), 161.

[AgGMV] L. Alvarez-Gaumé, P. Ginsparg, G. Moore, and C. Vafa, An $\mathrm{O}(16) \times \mathrm{O}(16)$ heterotic string, Phys. Lett. B 171 (1986), no. 2-3, 155-162.

[AgMV] Luis Àlvarez-Gaumé, Gregory W. Moore, and Cumrun Vafa, Theta functions, modular invariance, and strings, Commun. Math. Phys. 106 (1986), $1-40$.

[ALR] Alejandro Adem, Johann Leida, and Yongbin Ruan, Orbifolds and stringy topology, Cambridge Tracts in Mathematics, vol. 171, Cambridge University Press, Cambridge, 2007.

[AS] M. F. Atiyah and G. B. Segal, Equivariant K-theory and completion, J. Differential Geometry 3 (1969), 1-18.

[AW] Joseph J. Atick and Edward Witten, The Hagedorn Transition and the Number of Degrees of Freedom of String Theory, Nucl. Phys. B310 (1988), 291-334.

[B] M. Basterra, André-Quillen cohomology of commutative S-algebras, J. Pure Appl. Algebra 144 (1999), no. 2, 111-143.

[Br] Jean-Luc Brylinski, Loop Spaces, Characteristic Classes and Geometric Quantization, Progress in Mathematics, vol. 107, Birkhäuser, Boston, MA, 1993.

$15_{\text {generated }}$ by the worldsheet transformations $(-1)^{F_{L}},(-1)^{F_{R}}$ and worldsheet parity $\Omega$ 
[Bro] Edgar H. Brown, Jr., Generalizations of the Kervaire invariant, Ann. of Math. (2) 95 (1972), 368-383.

[Bu] U. Bunke, String structures and trivialisations of a pfaffian line bundle, arXiv:0909.0846.

[CS] Jeff Cheeger and James Simons, Differential characters and geometric invariants, Geometry and topology (College Park, Md., 1983/84), Lecture Notes in Math., vol. 1167, Springer, Berlin, 1985, pp. 50-80.

[De] Pierre Deligne, Notes on spinors, Quantum Fields and Strings: a course for mathematicians, Vol. 1, 2 (Princeton, NJ, 1996/1997), Amer. Math. Soc., Providence, RI, 1999, pp. 99-135.

[DF] Pierre Deligne and Daniel S. Freed, Classical field theory, Quantum Fields and Strings: a course for mathematicians, Vol. 1, 2 (Princeton, NJ, 1996/1997), Amer. Math. Soc., Providence, RI, 1999, pp. 137-225.

[DFM1] Jacques Distler, Daniel S. Freed, and Gregory W. Moore, Orientifold precis, in "Mathematical Foundations of Quantum Field Theory and Perturbative String Theory," B. Jurco, H. Sati, U. Schreiber, eds., American Mathematical Society, to appear, arXiv:0906.0795 [hep-th].

[DFM2] in preparation.

[DFM3] . in preparation.

$[\mathrm{DH}]$

[DHVW] Lance J. Dixon, Jeffrey A. Harvey, C. Vafa, and Edward Witten, Strings on orbifolds, Nucl. Phys. B261 (1985), 678-686.

[F1] Daniel S. Freed, K-theory in quantum field theory, Current developments in mathematics, 2001, Int. Press, Somerville, MA, 2002, pp. 41-87. math-ph/0206031.

[F2] On determinant line bundles, Mathematical Aspects of String Theory (S. T. Yau, ed.), Advanced Series in Mathematical Physics, vol. 1, 1986, pp. 189-238.

[FH] Daniel S. Freed and Michael J. Hopkins, On Ramond-Ramond fields and K-theory, JHEP 05 (2000), 044, arXiv:hep-th/0002027.

[FHT] D. S. Freed, M. J. Hopkins, and C. Teleman, Loop groups and twisted Ktheory I, J. Topology, 0711.1906. to appear.

[FMS] Daniel S. Freed, Gregory W. Moore, and Graeme Segal, The uncertainty of fluxes, Commun. Math. Phys. 271 (2007), 247-274, arXiv:hep-th/ 0605198

[FW] D. Friedan and Paul Windey, Supersymmetric derivation of the AtiyahSinger index and the chiral anomaly, Nucl. Phys. B235 (1984), 395.

[G] Kiyonori Gomi, Equivariant smooth Deligne cohomology, Osaka J. Math. 42 (2005), no. 2, 309-337, math/0307373.

[GHM] Michael B. Green, Jeffrey A. Harvey, and Gregory W. Moore, I-brane inflow and anomalous couplings on D-branes, Class. Quant. Grav. 14 (1997), 47-52, arXiv: hep-th/9605033.

[GSO1] F. Gliozzi, J. Scherk, and D. Olive, Supergravity and the spinor dual model, Physics Letters B 65 (1976), no. 3, 282-286.

[GSO2] Supersymmetry, supergravity theories and the dual spinor model, Nuclear Physics B 122 (1977), no. 2, 253-290.

[GSW] Krzysztof Gawedzki, Rafal R. Suszek, and Konrad Waldorf, Bundle gerbes for orientifold sigma models, arXiv:0809.5125 [math-ph].

[Hi] Nigel Hitchin, Lectures on special Lagrangian submanifolds, Winter School on Mirror Symmetry, Vector Bundles and Lagrangian Submanifolds 
(Cambridge, MA, 1999), AMS/IP Stud. Adv. Math., vol. 23, Amer. Math. Soc., Providence, RI, 2001, pp. 151-182. math/9907034.

[HS] M. J. Hopkins and I. M. Singer, Quadratic functions in geometry, topology, and M-theory, J. Diff. Geom. 70 (2005), 329-452, arXiv:math/0211216.

[KT] R. C. Kirby and L. R. Taylor, Pin structures on low-dimensional manifolds, Geometry of Low-Dimensional Manifolds, 2 (Durham, 1989), London Math. Soc. Lecture Note Ser., vol. 151, Cambridge Univ. Press, Cambridge, 1990, pp. 177-242.

[LU] Ernesto Lupercio and Bernardo Uribe, Deligne cohomology for orbifolds, discrete torsion and B-fields, Geometric and Topological Methods for Quantum Field Theory (Villa de Leyva, 2001), World Sci. Publ., River Edge, NJ, 2003, pp. 468-482. hep-th/0201184.

[M] Gregory W. Moore, K-theory from a physical perspective, arXiv:hep-th/0304018.

[MM] Ruben Minasian and Gregory W. Moore, K-theory and Ramond-Ramond charge, JHEP 11 (1997), 002, arXiv: hep-th/9710230.

[MS] J. P. May and J. Sigurdsson, Parametrized Homotopy Theory, Mathematical Surveys and Monographs, vol. 132, American Mathematical Society, Providence, RI, 2006.

[Mu $\quad$ M. K. Murray, Bundle gerbes, J. London Math. Soc. (2) 54 (1996), no. 2, 403-416.

[MW] Gregory W. Moore and Edward Witten, Self-duality, Ramond-Ramond fields, and K-theory, JHEP 05 (2000), 032, arXiv:hep-th/9912279.

[OS] Kasper Olsen and Richard J. Szabo, Constructing D-Branes From KTheory, Adv. Theor. Math. Phys. 3 (1999), 889-1025, arXiv:hep-th/ 9907140.

[R] Ryan Rohm, Spontaneous supersymmetry breaking in supersymmetric string theories, Nuclear Physics B 237 (1984), no. 3, 553-572.

[RW] R. Rohm and Edward Witten, The antisymmetric tensor field in superstring theory, Ann. Phys. 170 (1986), 454.

[S] I. M. Singer, The geometric interpretation of a special connection, Pacific J. Math. 9 (1959), 585-590.

[Sa] I. Satake, On a generalization of the notion of manifold, Proc. Nat. Acad. Sci. U.S.A. 42 (1956), 359-363.

[Se] Graeme Segal, Categories and cohomology theories, Topology 13 (1974), 293-312.

[Sh] Eric Sharpe, Discrete torsion, Phys. Rev. D (3) 68 (2003), no. 12, 126003, 20.

[SS1] Joel Scherk and John H. Schwarz, Spontaneous breaking of supersymmetry through dimensional reduction, Phys. Lett. B82 (1979), 60.

[SS2] How to get masses from extra dimensions, Nucl. Phys. B153 (1979), $61-88$.

[SW] Nathan Seiberg and Edward Witten, Spin structures in string theory, Nucl. Phys. B276 (1986), 272.

[Th] William P. Thurston, Three-dimensional Geometry and Topology. Vol. 1, Princeton Mathematical Series, vol. 35, Princeton University Press, Princeton, NJ, 1997. Edited by Silvio Levy.

[W1] Edward Witten, Index of Dirac operators, Quantum Fields and Strings: a course for mathematicians, Vol. 1, 2 (Princeton, NJ, 1996/1997), Amer. Math. Soc., Providence, RI, 1999, pp. 475-512.

[W2] D-branes and K-theory, JHEP 12 (1998), 019, arXiv:hep-th/ 9810188 
[W3]

Overview of K-theory applied to strings, Int. J. Mod. Phys. A16 (2001), 693-706, arXiv:hep-th/0007175.

[Wa] C. T. C. Wall, Graded Brauer groups, J. Reine Angew. Math. 213 (1963/1964), 187-199.

Theory Group, Department of Physics, and Texas Cosmology Center, UniVersity of Texas, 1 University Station C1600, Austin, TX 78712-0264

E-mail address: distler@golem.ph.utexas.edu

Department of Mathematics, University of Texas, 1 University Station C1200, Austin, TX 78712-0257

E-mail address: dafr@math.utexas.edu

NHetC and Department of Physics and Astronomy, Rutgers University, PISCATAWAY, NJ 08855-0849

E-mail address: gmoore@physics.rutgers.edu 